\title{
Application of magnetic resonance technologies in aquatic biology and seafood science
}

\author{
Gen Kaneko ${ }^{1}\left(\mathbb{D} \cdot\right.$ Hideki Ushio $^{2} \cdot{\text { Hong } \mathrm{Ji}^{3}}^{3}$
}

Received: 31 July 2018 / Accepted: 16 October 2018 / Published online: 9 November 2018

(c) The Author(s) 2018

\begin{abstract}
Magnetic resonance technologies, such as nuclear magnetic resonance (NMR) spectroscopy and magnetic resonance imaging (MRI), are powerful tools used in various fields of science. In this review, we provide a broad overview of the application of magnetic resonance technologies in aquatic biology following brief descriptions of their principles and characteristics. The topics covered include in vitro and in vivo NMR spectroscopy, NMR metabolomics, MRI, and their application to seafood science. Special attention is paid to in vivo NMR spectroscopy and metabolic tracing using a stable isotope, ${ }^{13} \mathrm{C}$, which provide safe and effective means of exploring the metabolic diversity of aquatic organisms.
\end{abstract}

Keywords In vivo nuclear magnetic resonance spectroscopy $\cdot$ Metabolic flux $\cdot$ Magnetic resonance imaging $\cdot$ Stable isotope

\section{Introduction}

Certain nuclei have magnetic moments that arise from the spin of protons and neutrons. Such nuclei are called magnetic nuclei, and biologically important magnetic nuclei include ${ }^{1} \mathrm{H},{ }^{13} \mathrm{C},{ }^{14} \mathrm{~N},{ }^{15} \mathrm{~N},{ }^{17} \mathrm{O},{ }^{19} \mathrm{~F},{ }^{23} \mathrm{Na}$, and ${ }^{31} \mathrm{P}$. Their magnetic moments are randomly oriented in the absence of an external magnetic field, the strength of which is usually represented by $B_{0}$ (i.e., in this case $B_{0}=0$ ). However, when magnetic moments are placed in a high $B_{0}$ field, they are predominantly oriented either parallel or antiparallel to

Japan Society for the Promotion of Science (JSPS) Grant-in-Aid for Scientific Research (KAKENHI) no. JP 262003.

Gen Kaneko

kanekog@uhv.edu

Hideki Ushio

aushio@mail.ecc.u-tokyo.ac.jp

Hong Ji

jihong@nwsuaf.edu.cn

1 School of Arts and Sciences, University of Houston-Victoria, 3007, North Ben Wilson, Victoria, TX 77901, USA

2 Department of Aquatic Bioscience, Graduate School of Agricultural and Life Sciences, The University of Tokyo, Yayoi, Bunkyo, Tokyo 113-8657, Japan

3 College of Animal Science and Technology, Northwest A\&F University, Yangling 712100, People's Republic of China the applied field (Keeler 2010; Kemp 1986). The aligned magnetic moments flip their orientation when they absorb or emit electromagnetic waves at a specific frequency [resonance frequency $(v)$ or Larmor frequency]. This phenomenon, termed nuclear magnetic resonance (NMR), was first identified in 1938 using a beam of lithium chloride molecules passing through a vacuum chamber (Rabi et al. 1939). A few years later, two groups independently detected NMR in solid and liquid substances (Bloch et al. 1946; Purcell et al. 1946). Three authors of these papers were awarded the Nobel Prize in Physics for the discovery of NMR in 1944 and 1952. The discovery of NMR led to the development of NMR spectroscopy, in which absorption features of electromagnetic waves, mainly the frequency and amount, provide valuable information about molecules. NMR spectroscopy further triggered the development of various magnetic resonance technologies [e.g., in vivo NMR and magnetic resonance imaging (MRI)] that have been used as powerful tools in physics, chemistry, and biology. In this section, we briefly introduce the principle of NMR. Although NMR spectroscopy is often used as an example, the principle can be applied to all magnetic resonance technologies described in this review.

The resonance frequency $v_{0}$ is a function of $B_{0}$ and the gyromagnetic ratio $(\gamma)$, a constant parameter for a particular nuclear type (Eq. 1). Each magnetic nucleus therefore has a specific resonance frequency. For example, in an external magnetic field of $11.7 \mathrm{~T}$, the resonance frequency of ${ }^{1} \mathrm{H}$ is 
approximately $500 \mathrm{MHz}$, whereas that of ${ }^{13} \mathrm{C}$ is approximately $125 \mathrm{MHz}$.

$v_{0}=\left(\frac{\gamma}{2 \pi}\right) B_{0}$

What makes magnetic resonance technologies powerful tools in science is the finding that the resonance frequency $v$ is affected by the local chemical environment of the nuclei of interest (Arnold et al. 1951). Namely, electrons surrounding the nuclei of interest produce a local magnetic field, and therefore the actual magnetic field sensed by the nuclei of interest is represented as $B_{0}(1-\sigma)$, where $\sigma$ is a dimensionless number called the shielding constant (Eq. 2). The actual $v$ is thus different from $v_{0}$ in Eq. 1 .

$v=\left(\frac{\gamma}{2 \pi}\right) B_{0}(1-\sigma)$

The Eq. 2 shows that, for example, ${ }^{1} \mathrm{H}$ in different chemical groups, such as $-\mathrm{CH}_{3}$ and $-\mathrm{CHO}$, resonate at slightly

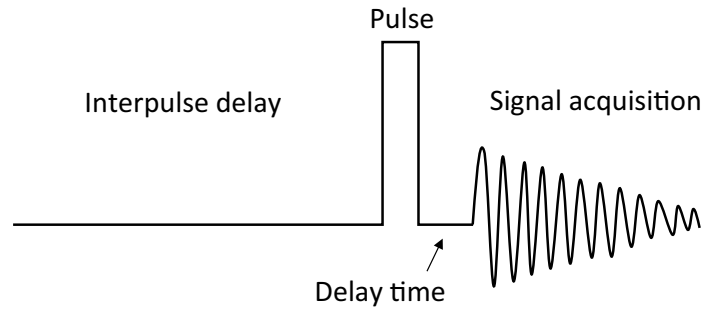

Fig. 1 Schematic representation of a simple pulse sequence used in ${ }^{1} \mathrm{H}$ nuclear magnetic resonance (NMR) spectroscopy. The radio-frequency pulse affects the orientation of magnetic moments in samples, resulting in the oscillation voltage in a detector coil (NMR signal). This signal is called the free induction decay (FID) since it decays as the magnetic moments return to equilibrium after the end of the pulse. Spectra from modern NMR systems are Fourier-transformed FID signals different frequencies reflecting the difference in their chemical structures. Hence, determination of the resonance frequency by an NMR spectrometer provides structural information of the target molecule. Modern magnetic resonance systems (e.g., NMR spectrometers and MRI scanners) use a radiofrequency pulse containing multiple frequency components, and thus many magnetic nuclei having different resonance frequencies resonate at the same time. The setting parameters to acquire NMR signals are called the pulse sequence (Fig. 1).

In this review, we summarize the contribution of magnetic resonance technologies to aquatic biology and seafood science, and discuss future uses for these technologies in the era of multiomics approaches. Special attention is paid to in vivo NMR spectroscopy and metabolic tracing, which provide safe and effective means of exploring the metabolic diversity of aquatic organisms.

\section{NMR spectroscopy}

\section{Principle and characteristics}

In vitro NMR spectroscopy achieves higher sensitivity and spectral resolution compared to in vivo NMR spectroscopy (Table 1), and has been the first choice for most analytical purposes. Table 2 summarizes the characteristics of NMR spectroscopy for different magnetic nuclei. ${ }^{1} \mathrm{H}$ NMR has been the most frequently used NMR technique because of its high sensitivity (both in terms of high gyrometric ratio and natural abundance of ${ }^{1} \mathrm{H}$ ). Another advantage of ${ }^{1} \mathrm{H}$ NMR spectroscopy is that most metabolites contain ${ }^{1} \mathrm{H}$. Although most metabolites also contain carbon, the low natural abundance of ${ }^{13} \mathrm{C}$ results in the low sensitivity of ${ }^{13} \mathrm{C}$ NMR spectroscopy. On the other hand, ${ }^{31} \mathrm{P}$ NMR spectroscopy has been a key technology for understanding energy states

Table 1 Comparison of magnetic resonance technologies

\begin{tabular}{llll}
\hline Technology & Benefits & Weakness & Major application \\
\hline NMR spectroscopy (in vitro) & High sensitivity and resolution & Invasive & $\begin{array}{c}\text { Structure determination and } \\
\text { metabolomics }\end{array}$ \\
NMR spectroscopy (in vivo) & $\begin{array}{c}\text { Non-invasive real-time quantifica- } \\
\text { tion of metabolites }\end{array}$ & $\begin{array}{c}\text { Lower sensitivity and resolution } \\
\text { compared to in vitro }\end{array}$ & $\begin{array}{c}\text { In vivo monitoring of metabolites; } \\
\text { especially useful for ATP-related } \\
\text { compounds }\end{array}$ \\
$\begin{array}{l}\text { Stable isotope tracing (in vitro, } \\
\text { in vivo) }\end{array}$ & $\begin{array}{c}\text { Specific quantification of labeled } \\
\text { substances }\end{array}$ & $\begin{array}{c}\text { High cost of stable isotope- } \\
\text { labeled substance }\end{array}$ & $\begin{array}{c}\text { Quantitative exploration of meta- } \\
\text { bolic pathways; few applications } \\
\text { in aquatic biology }\end{array}$ \\
$\begin{array}{l}\text { Structural MRI } \\
\text { Functional MRI }\end{array}$ & $\begin{array}{c}\text { Non-invasive visualization of vari- } \\
\text { ous tissues } \\
\text { Non-invasive visualization of } \\
\text { blood oxygenation parameters }\end{array}$ & $\begin{array}{c}\text { Low resolution compared to CT } \\
\text { fimited target tissues (typically }\end{array}$ & $\begin{array}{c}\text { Food science and physiological } \\
\text { studies }\end{array}$ \\
bew applications in aquatic biology
\end{tabular}

NMR Nuclear magnetic resonance, MRI magnetic resonance imaging, $C T$ computed tomography 
Table 2 Comparison of NMR using different magnetic nuclei

\begin{tabular}{|c|c|c|c|c|}
\hline Technology & Major target & $\begin{array}{l}\text { Natural abundance } \\
\text { of target nuclei }(\%)\end{array}$ & Relative sensitivity $^{a}$ & Other notes \\
\hline${ }^{1} \mathrm{H}$ NMR & Most endogenous metabolites & 100.0 & 1.00 & $\begin{array}{l}\text { Common NMR modality despite its narrow chemical } \\
\text { shift range }\end{array}$ \\
\hline${ }^{2} \mathrm{H}$ NMR & ${ }^{2} \mathrm{H}$-labeled molecules (tracer) & 0.02 & $1.45 \times 10^{-6}$ & Not common in aquatic biology due to low sensitivity \\
\hline${ }^{13} \mathrm{C}$ NMR & $\begin{array}{l}\text { Most endogenous metabo- } \\
\text { lites, }{ }^{13} \mathrm{C} \text {-labeled molecules } \\
\text { (tracer) }\end{array}$ & 1.1 & $1.76 \times 10^{-4}$ & Common NMR modality despite its low sensitivity \\
\hline${ }^{14} \mathrm{~N} N M R$ & Amines & 99.6 & $1.00 \times 10^{-3}$ & Signals are broad due to quadrupolar interactions \\
\hline${ }^{15} \mathrm{~N} N M R$ & ${ }^{15} \mathrm{~N}$-labeled molecules (tracer) & 0.4 & $3.86 \times 10^{-6}$ & Requires enrichment due to low sensitivity \\
\hline${ }^{23} \mathrm{Na} \mathrm{NMR}$ & Sodium salts & 100.0 & $9.27 \times 10^{-2}$ & MRI has been used for fish fillets \\
\hline${ }^{31} \mathrm{P}$ NMR & $\begin{array}{l}\text { ATP-related compounds, } \\
\text { phosphocreatine (PCr), } \\
\text { intracellular } \mathrm{pH}\end{array}$ & 100.0 & $6.65 \times 10^{-2}$ & Limited number of target molecules \\
\hline
\end{tabular}

For abbreviations, see Table 1

${ }^{\mathrm{a}}$ Cited from de Graaf (2007)

because it is able to directly quantify levels of phosphocreatine (PCr), ATP, and inorganic phosphate (Pi). Furthermore, the difference between chemical shifts of $\mathrm{PCr}$ and $\mathrm{Pi}$ allows the determination of intracellular $\mathrm{pH}$ (Moon and Richards 1973), which is affected by the accumulation of lactate, the final product of anaerobic glycolysis. Other NMR technologies will be mentioned later with specific examples.

In NMR spectroscopy, signals from multiple magnetic nuclei are Fourier transformed and generally represented as an NMR spectrum (Fig. 2). The $x$-axis is the small observed change in resonance frequency, called the chemical shift $(\delta)$, which is usually expressed in parts per million ( $\mathrm{ppm}$ ) according to frequency using reference compounds (Keeler 2010). Tetramethylsilane is a common reference compound in ${ }^{1} \mathrm{H}$ NMR spectroscopy using organic solvents, whereas trimethylsilyl propanoic acid and 4,4-dimethyl-4-silapentane-1-sulfonic acid (DSS) are used as reference compounds in aqueous solvents. For example, ${ }^{1} \mathrm{H}$ resonance frequencies of molecules shown in Fig. 2 are 1.0-2.3 ppm higher than those of the reference proton of DSS. Notation in parts per million is useful to represent the small effects of the local chemical environment on resonance frequency. Furthermore, the chemical shift according to this definition is a relative, dimensionless parameter independent of the applied magnetic field. NMR spectrometers with different magnetic field strength therefore give the same chemical shift value for the same nucleus, enabling interlaboratory comparison of NMR spectra.

NMR spectroscopy is also a useful tool for quantitative NMR metabolomics, pioneered by Nicholson et al. (1984, 1983), because the signal intensity in NMR spectroscopy is proportional to the number of nuclei (Kemp 1986). Among various NMR techniques, ${ }^{1} \mathrm{H}$ NMR has been the predominant method for metabolomics due to its high sensitivity and

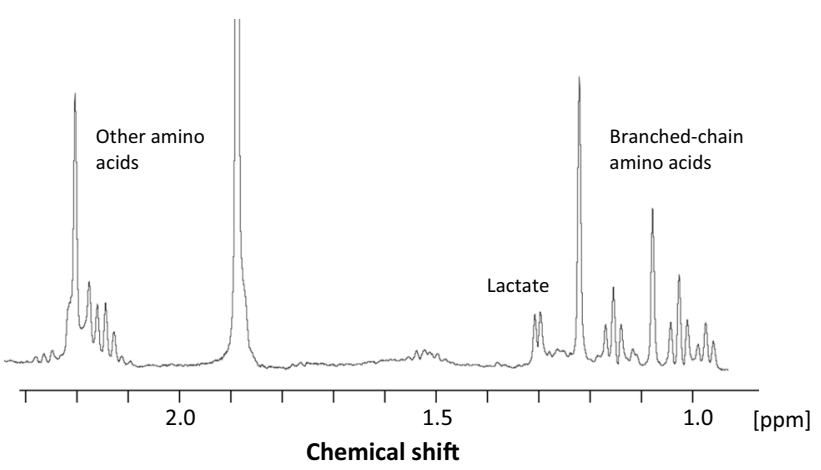

Fig. 2 Typical ${ }^{1} \mathrm{H}$ NMR spectrum obtained from an aqueous extract of the rotifer Brachionus manjavacas. Putative peak assignment is shown. Rotifer extracts were prepared from approximately $10 \mathrm{mg}$ (wet weight) of fed B. manjavacas according to a previous report (Behar et al. 1983) with slight modifications. Briefly, rotifer tissues were homogenized in $200 \mu \mathrm{L}$ of $0.1 \mathrm{M} \mathrm{HCl} /$ methanol. The homogenate was added to $600 \mu \mathrm{L}$ of an ice-cold 9:1 mixture of ethanol and $100 \mathrm{mM}$ potassium-phosphate buffer ( $\mathrm{pH} 7.4$ ), and incubated for $15 \mathrm{~min}$ on ice with occasional mixing. After addition of $600 \mu \mathrm{L}$ of distilled water, the mixture was centrifuged at $10,000 \mathrm{~g}$ for $10 \mathrm{~min}$. The supernatant was deionized by Chelex 100 Resin (Bio-Rad). The resulting extracts $(\sim 6 \mathrm{~mL})$ were lyophilized, dissolved in the $600 \mu \mathrm{L}$ of a 2:1 mixture of $\mathrm{D}_{2} \mathrm{O}$ and $100 \mathrm{mM}$ potassium phosphate buffer, and subjected to ${ }^{1} \mathrm{H}$ NMR using an 11.7-T Bruker vertical-bore spectrometer (Bruker, Billerica, MA). Spectra were analyzed by TopSpin 3.0 (Bruker). ppm parts per million

the prevalence of protons in biological systems (Table 2). Compared to mass spectrometry (MS), another common technology for metabolomics, ${ }^{1} \mathrm{H}$ NMR spectroscopy has at least two advantages in metabolomics studies (Beckonert et al. 2007). First, ${ }^{1} \mathrm{H}$ NMR spectroscopy is a non-destructive technique-the same sample can be scanned as many times as required. Because the signal-to-noise ratio of NMR 
spectra is proportional to the square root of the number of scans (i.e. $S / N \sim \sqrt{ } n$, where $n$ is the number of scans), averaging multiple scans results in improved $S / N$ ratios (Traficante 1991). Also, the same sample can be analyzed in many different pulse sequences optimized for the detection of specific metabolites. Secondly, NMR spectroscopy requires less sample preparation compared to MS. Blood, serum, plasma, and urine samples are commonly used in human studies without prior chromatographic separation (Beckonert et al. 2007). Urine in particular requires minimal sample preparation because it contains low amounts of macromolecules (e.g., proteins and lipids) that distort the NMR spectra baseline. Other biological fluids such as cerebrospinal fluid and saliva have also been analyzed by NMR metabolomics (Bollard et al. 2005).

The major limitation of NMR metabolomics is the low sensitivity compared to MS. Even after repeated scans, the detection limit of NMR is still in the order of micromols per gram wet tissue in general (Table 3). Also, some pretreatments are required for samples of high ionic strength (Beckonert et al. 2007). Thus NMR and MS have different advantages, and their synergistic use is the best practice for global metabolic profiling.

\section{Structure determination by NMR in aquatic biology}

Aquatic organisms are a rich source of natural compounds with various biological activities ( $\mathrm{Li}$ et al. 2015). Although many current drugs derived from natural compounds have terrestrial origin (Cragg and Newman 2001), this is probably due to the practical difficulty in collecting samples from aquatic environments. There has been, and will be, numerous attempts to discover potential drugs from aquatic organisms, especially marine invertebrates, because of the diversity and strong biological activity of compounds from them. ${ }^{1} \mathrm{H}$ and ${ }^{13} \mathrm{C}$ NMR spectroscopy have played important roles in determining the structure of biologically active natural compounds.

One of the pioneering works in this field is the discovery of pseudopterosins from Pseudopterogorgia elisabethae (Fenical 1987; Look et al. 1986). P. elisabethae is a cnidarian found in Caribbean coastal waters. Analyses demonstrated that this species contains high amounts of polar compounds with potent anti-inflammatory activity (Fenical 1987; Look et al. 1986). Look et al. (1986) identified four novel glycosides from $P$. elisabethae by a combination of NMR, X-ray crystallography, and MS, and termed these compounds pseudopterosins A-D. Pseudopterosins are now commercially used in skin creams as anti-inflammatory agents. Another successful example is the identification of norhalichondrin A from the sponge Halichondria okadai (Uemura et al. 1985), which led to the synthesis of anticancer drug E7389 (Kuznetsov et al. 2004). It is beyond the scope of this review to provide a comprehensive overview of NMR-based structure determination. Readers can refer to specialized reviews for studies on marine sponges (Lira et al. 2011; Takada 2015), invertebrate glycosaminoglycans (Pomin 2015), and the dereplication strategy (Pérez-Victoria et al. 2016).

Notable developments in this field during the past decade include the significant improvement of sensitivity and combination with omics technologies. Novel probes and cryogenically cooled coils achieved a 20 -fold increase in sensitivity at the same magnetic field, enabling the identification of natural compounds at the nanomole scale (Molinski 2010). The highly sensitive technique of NMR spectroscopy has facilitated the characterization of secondary metabolites, which are not directly involved in vital metabolism and thus often found in low concentrations. Several studies have elucidated the structure of secondary metabolites by NMR spectroscopy in marine bacteria after prediction of their existence by genomic analyses (Paulus et al. 2017; Udwary et al. 2007). This new technological endeavor will aid future identification of novel natural compounds from aquatic metazoans.

\section{NMR metabolomics in aquatic biology}

There is an increasing number of ${ }^{1} \mathrm{H}$ NMR metabolomics studies on aquatic organisms. While many studies have used 500-600 MHz spectrometers, some have taken advantage of higher magnetic fields of $16.4 \mathrm{~T}(700 \mathrm{MHz})$ (Cappello et al. 2013; Lardon et al. 2013) and $18.8 \mathrm{~T}$ (800 MHz) (Ekman et al. 2007; Southam et al. 2008). The most commonly used samples are plasma and tissue extracts of muscle, brain, and liver. Urine has barely been used for fish NMR metabolomics since Ekman et al. (2007). Liquid nitrogen has been commonly used for quick freezing of tissues for extraction of metabolites. However, aqueous metabolites include ATP, $\mathrm{ADP}$, and lactate, the amount of which rapidly changes during post-mortem extraction even when procedures are carried out carefully. Microwave fixation is an alternative method for this (de Graaf et al. 2009). The methanol-chloroform (M/C) method has been shown to be superior to the perchloric acid method for mammalian tissues because it can extract both aqueous and lipophilic metabolites from a single sample with lower variability (Le Belle et al. 2002). The M/C method has been optimized for fish samples by Lin et al. (2007) and Wu et al. (2008).

Table 3 shows chemical shift values and concentration ranges of major aqueous metabolites identified from aquatic organisms by NMR metabolomics. The amounts of the metabolites are, unfortunately, mainly represented by metabolite concentrations (millimols) in sample solutions or merely as fold changes in the literature, and concentration ranges of these metabolites in Table 3 are mostly 
Table 3 Chemical shift and concentration range of representative metabolites in aquatic organisms that can be identified by NMR

\begin{tabular}{|c|c|c|c|c|}
\hline \multirow[t]{2}{*}{ Molecule } & \multirow{2}{*}{$\begin{array}{l}\text { NMR information } \\
\text { Chemical shift in } \mathrm{H}_{2} \mathrm{O}(\mathrm{ppm})^{\mathrm{a}}\end{array}$} & \multicolumn{3}{|c|}{ Biological information } \\
\hline & & Tissue & $\begin{array}{l}\text { Concentration ( } \mu \mathrm{mol} / \mathrm{g} \text { wet } \\
\text { tissue; } \mu \mathrm{mol} / \mathrm{mL} \text { for plasma } \\
\text { and blood) }\end{array}$ & Organism and reference $^{b}$ \\
\hline \multicolumn{5}{|c|}{ Amino acids and their derivatives } \\
\hline \multirow[t]{3}{*}{ Glycine } & \multirow[t]{3}{*}{$3.54\left(\mathrm{~s},{ }^{2} \mathrm{CH}_{2}\right)$} & Brain & $0.82-1.98 / 2.3$ & O. mykiss ${ }^{\mathrm{A}} / O$. mykiss $^{\mathrm{D}}$ \\
\hline & & Muscle & $1.35-12.81 / 18.1-29.4$ & P. lethostigma ${ }^{\mathrm{B}} / O \cdot$ mordax $^{\mathrm{G}}$ \\
\hline & & Plasma & $0.5-1.3 / 0.37-0.45$ & O. mykiss ${ }^{\mathrm{A}} / S$. salar $\mathrm{V}$ \\
\hline \multirow[t]{3}{*}{ Alanine } & \multirow[t]{3}{*}{$3.76\left(\mathrm{~m},{ }^{2} \mathrm{CH}\right), 1.46\left(\mathrm{~d},{ }^{3} \mathrm{CH}_{3}\right)$} & Brain & $0.33-0.38 / 0.67 / 0.74$ & $\begin{array}{l}\text { O. mykiss }{ }^{\mathrm{A}} / O . \text { mykiss }^{\mathrm{D}} / D . \\
\text { rario }^{\mathrm{I}}\end{array}$ \\
\hline & & Muscle & $0.93-5.10 / 6.2-9.7$ & P. lethostigma ${ }^{\mathrm{B}} / O \cdot$ mordax $^{\mathrm{G}}$ \\
\hline & & Plasma & $0.27-0.48$ & O. mykiss $^{\mathrm{A}}$ \\
\hline \multirow[t]{3}{*}{ Glutamate } & \multirow[t]{3}{*}{$\begin{array}{l}3.74\left(\mathrm{t},{ }^{2} \mathrm{CH}\right), 2.12(\mathrm{~m}, \\
\left.{ }^{3} \mathrm{CH}_{2}\right), 2.34\left(\mathrm{~m},{ }^{4} \mathrm{CH}_{2}\right)\end{array}$} & Brain & $5.6-6.1 / 17.9 / 5.82$ & $\begin{array}{l}\text { O. mykiss }{ }^{\mathrm{A}} / O . \text { mykiss }^{\mathrm{D}} / D . \\
\text { rario }^{\mathrm{I}}\end{array}$ \\
\hline & & Muscle & $0.40-1.80 / 0.38-1.26$ & P. lethostigma ${ }^{\mathrm{B}} / O \cdot$ mordax $^{\mathrm{G}}$ \\
\hline & & Plasma & $0.04-0.10$ & O. mykiss ${ }^{\mathrm{A}}$ \\
\hline \multirow[t]{3}{*}{ Glutamine } & \multirow{3}{*}{$\begin{array}{l}3.75\left(\mathrm{t},{ }^{2} \mathrm{CH}\right), 2.08(\mathrm{~m}, \\
\left.{ }^{3} \mathrm{CH}_{2}\right), 2.43\left(\mathrm{~m},{ }^{4} \mathrm{CH}_{2}\right)\end{array}$} & Brain & $3.8-4.2 / \sim 3$ & O. mykiss ${ }^{\mathrm{A}} / C$. auratus $\mathrm{C}^{\mathrm{C}}$ \\
\hline & & Muscle & $0.18-0.43$ & O. $\operatorname{mordax}^{\mathrm{G}}$ \\
\hline & & Plasma & $0.22-0.45$ & O. $m y k i s s^{\mathrm{A}}$ \\
\hline \multirow[t]{3}{*}{ Aspartate } & \multirow[t]{3}{*}{$\begin{array}{l}3.89\left(\mathrm{dd},{ }^{2} \mathrm{CH}\right), 2.80(\mathrm{dd}, \\
\left.{ }^{3} \mathrm{CH}_{2}\right), 2.65\left(\mathrm{dd},{ }^{3} \mathrm{CH}_{2}\right)\end{array}$} & Brain & $0.70-1.05 / 7.5 / 1.13$ & $\begin{array}{l}\text { O. mykiss }{ }^{\mathrm{A}} / O . \text { mykiss }^{\mathrm{D}} / D \text {. } \\
\text { rario }^{\mathrm{I}}\end{array}$ \\
\hline & & Muscle & $0.16-1.66 / \sim 1.15$ & P. lethostigma ${ }^{\mathrm{B}} / O \cdot$ mordax $^{\mathrm{G}}$ \\
\hline & & Plasma & $<0.01$ & O. mykiss ${ }^{\mathrm{A}}$ \\
\hline \multirow[t]{3}{*}{ Asparagine } & \multirow{3}{*}{$\begin{array}{l}4.00\left(\mathrm{dd},{ }^{2} \mathrm{CH}\right), 2.94(\mathrm{~m}, \\
\left.{ }^{3} \mathrm{CH}_{2}\right), 2.84\left(\mathrm{~m},{ }^{3} \mathrm{CH}_{2}\right)\end{array}$} & Brain & ND & O. mykiss ${ }^{\mathrm{A}}$ \\
\hline & & Muscle & $0.15-0.29$ & O. $\operatorname{mordax}^{\mathrm{G}}$ \\
\hline & & Plasma & $0.13-0.21$ & O. mykiss ${ }^{\mathrm{A}}$ \\
\hline \multirow[t]{3}{*}{ Valine } & \multirow{3}{*}{$\begin{array}{l}3.59\left(\mathrm{~d},{ }^{2} \mathrm{CH}\right), 2.25\left(\mathrm{~m},{ }^{3} \mathrm{CH}\right) \\
1.03\left(\mathrm{~d},{ }^{4} \mathrm{CH}_{3}\right), 0.98(\mathrm{~d} \\
\left.{ }^{4} \mathrm{CH}_{3}\right)\end{array}$} & Brain & $\sim 0.11$ & O. mykiss ${ }^{\mathrm{A}}$ \\
\hline & & Muscle & $0.31-0.38 / 0.26-0.54$ & P. lethostigma $/ O$. mordax $^{\mathrm{G}}$ \\
\hline & & Plasma & $0.43-0.60$ & O. mykiss ${ }^{\mathrm{A}}$ \\
\hline \multirow[t]{3}{*}{ Leucine } & \multirow{3}{*}{$\begin{array}{l}3.71\left(\mathrm{t},{ }^{2} \mathrm{CH}\right), 1.70\left(\mathrm{~m},{ }^{3} \mathrm{CH}_{2},\right. \\
\left.{ }^{4} \mathrm{CH}\right), 0.95\left(\mathrm{~m},{ }^{5} \mathrm{CH}_{3},\right. \\
\left.{ }^{5} \mathrm{CH}_{3}\right)\end{array}$} & Brain & $0.11-0.14$ & O. mykiss $^{\mathrm{A}}$ \\
\hline & & Muscle & $0.24-0.33 / 0.31-0.69$ & P. lethostigma ${ }^{\mathrm{B}} / O \cdot$ mordax $^{\mathrm{G}}$ \\
\hline & & Plasma & $0.35-0.50$ & O. mykiss $\mathrm{A}^{\mathrm{A}}$ \\
\hline \multirow[t]{3}{*}{ Isoleucine } & \multirow{3}{*}{$\begin{array}{l}3.66\left(\mathrm{~d},{ }^{2} \mathrm{CH}\right), 1.96\left(\mathrm{~m},{ }^{3} \mathrm{CH}\right), \\
1.00\left(\mathrm{~d},{ }^{3} \mathrm{CH}_{3}\right), 1.44(\mathrm{~m}, \\
\left.{ }^{4} \mathrm{CH}_{2}\right), 1.26\left(\mathrm{~m},{ }^{4} \mathrm{CH}_{2}\right), 0.92 \\
\left(\mathrm{t},{ }^{5} \mathrm{CH}_{3}\right)\end{array}$} & Brain & $\sim 0.05$ & O. mykiss ${ }^{\mathrm{A}}$ \\
\hline & & Muscle & $0.19-0.27 / 0.18-0.35$ & P. lethostigma ${ }^{\mathrm{B}} / O \cdot$ mordax $^{\mathrm{G}}$ \\
\hline & & Plasma & $0.19-0.28$ & O. mykiss $\mathrm{A}^{\mathrm{A}}$ \\
\hline \multirow[t]{3}{*}{ Tyrosine } & \multirow{3}{*}{$\begin{array}{l}7.19\left(\mathrm{~m},{ }^{2} \mathrm{CH},{ }^{6} \mathrm{CH}\right), 6.89(\mathrm{~m}, \\
\left.{ }^{3} \mathrm{CH},{ }^{5} \mathrm{CH}\right), 3.93\left(\mathrm{dd},{ }^{\alpha} \mathrm{CH}\right), \\
3.19\left(\mathrm{dd},{ }^{\beta} \mathrm{CH}_{2}\right), 3.03(\mathrm{dd}, \\
\left.{ }^{\beta} \mathrm{CH}_{2}\right)\end{array}$} & Brain & $0.07-0.10$ & O. mykiss ${ }^{\mathrm{A}}$ \\
\hline & & Muscle & $0.16-0.38 / 0.21-0.49$ & P. lethostigma ${ }^{\mathrm{B}} / O \cdot$ mordax $^{\mathrm{G}}$ \\
\hline & & Plasma & $0.08-0.14$ & O. mykiss ${ }^{\mathrm{A}}$ \\
\hline Phenylalanine & $7.42\left(\mathrm{~m},{ }^{3} \mathrm{CH},{ }^{5} \mathrm{CH}\right), 7.36(\mathrm{~m}$ & Brain & $0.13-0.14$ & O. mykiss ${ }^{\mathrm{A}}$ \\
\hline & $\left.{ }^{4} \mathrm{CH}\right), 7.31\left(\mathrm{~m},{ }^{2} \mathrm{CH},{ }^{6} \mathrm{CH}\right)$ & Muscle & $0.14-0.23 / \sim 0.09$ & P. lethostigma ${ }^{\mathrm{B}} / O \cdot$ mordax $^{\mathrm{G}}$ \\
\hline & $\begin{array}{l}3.97\left(\mathrm{dd},{ }^{\alpha} \mathrm{CH}\right), 3.28(\mathrm{dd}, \\
\left.{ }^{\beta} \mathrm{CH}_{2}\right)\end{array}$ & Plasma & $0.14-0.19$ & O. mykiss ${ }^{\mathrm{A}}$ \\
\hline Proline & $4.12\left(\mathrm{dd},{ }^{2} \mathrm{CH}\right), 3.41(\mathrm{dt}$ & Brain & $0.15-0.18$ & O. mykiss ${ }^{\mathrm{A}}$ \\
\hline & $\left.{ }^{5} \mathrm{CH}_{2}\right), 3.33\left(\mathrm{dt},{ }^{5} \mathrm{CH}_{2}\right), 2.34$ & Muscle & $0.66-1.46 / 0.15-0.21$ & P. lethostigma $/ O$. mordax $^{\mathrm{G}}$ \\
\hline & $\begin{array}{l}\left(\mathrm{m},{ }^{5} \mathrm{CH}_{2}\right), 2.06\left(\mathrm{~m},{ }^{3} \mathrm{CH}_{2}\right) \\
1.99\left(\mathrm{~m},{ }^{4} \mathrm{CH}_{2}\right)\end{array}$ & Plasma & $0.09-0.33$ & O. mykiss ${ }^{\mathrm{A}}$ \\
\hline Serine & $3.98\left(\mathrm{dd},{ }^{3} \mathrm{CH}_{2}\right), 3.93(\mathrm{dd}$ & Brain & $0.40-0.60 / 0.42$ & O. mykiss $\mathrm{A}^{\mathrm{A}}$ O. mykiss ${ }^{\mathrm{D}}$ \\
\hline & $\left.{ }^{3} \mathrm{CH}_{2}\right), 3.83\left(\mathrm{dd},{ }^{2} \mathrm{CH}\right)$ & Muscle & $0.53-2.27 / 0.60-0.75$ & P. lethostigma ${ }^{\mathrm{B}} / O \cdot$ mordax $^{\mathrm{G}}$ \\
\hline & & Plasma & $0.13-0.29 / 0.16-0.17$ & O. mykiss ${ }^{\mathrm{A}} / S$. salar ${ }^{\mathrm{V}}$ \\
\hline Threonine & $4.24\left(\mathrm{~m},{ }^{3} \mathrm{CH}_{2}\right), 3.58(\mathrm{~d}$, & Brain & $0.40-0.75 / 0.71$ & O. mykiss $\mathrm{A}^{\mathrm{A}}$ O. mykiss $\mathrm{D}^{\mathrm{D}}$ \\
\hline & $\left.{ }^{2} \mathrm{CH}\right), 1.32\left(\mathrm{~d},{ }^{4} \mathrm{CH}_{3}\right)$ & Muscle & $0.41-0.64 / 1.11-1.32$ & P. lethostigma ${ }^{\mathrm{B}} / O \cdot$ mordax $^{\mathrm{G}}$ \\
\hline & & Plasma & $0.20-0.42$ & O. mykiss $^{\mathrm{A}}$ \\
\hline
\end{tabular}


Table 3 (continued)

\begin{tabular}{|c|c|c|c|c|}
\hline \multirow[t]{2}{*}{ Molecule } & \multirow{2}{*}{$\begin{array}{l}\text { NMR information } \\
\text { Chemical shift in } \mathrm{H}_{2} \mathrm{O}(\mathrm{ppm})^{\mathrm{a}}\end{array}$} & \multicolumn{3}{|c|}{ Biological information } \\
\hline & & Tissue & $\begin{array}{l}\text { Concentration ( } \mu \mathrm{mol} / \mathrm{g} \text { wet } \\
\text { tissue; } \mu \mathrm{mol} / \mathrm{mL} \text { for plasma } \\
\text { and blood) }\end{array}$ & Organism and reference ${ }^{b}$ \\
\hline \multirow[t]{3}{*}{ Methionine } & \multirow{3}{*}{$\begin{array}{l}3.85\left(\mathrm{dd},{ }^{2} \mathrm{CH}\right), 2.63(\mathrm{t}, \\
\left.{ }^{4} \mathrm{CH}_{2}\right), 2.12\left(\mathrm{~m},{ }^{3} \mathrm{CH}_{2},\right. \\
\left.{ }^{6} \mathrm{CH}_{3}\right)\end{array}$} & Brain & $0.04-0.08$ & O. mykiss ${ }^{\mathrm{A}}$ \\
\hline & & Muscle & $0.27-0.33 / 0.38-0.45$ & P. lethostigma ${ }^{\mathrm{B}} / O$. mordax $^{\mathrm{G}}$ \\
\hline & & Plasma & $0.07-0.31 / 0.10-0.31$ & O. mykiss $^{\mathrm{A}} / \mathrm{S}$. salar $^{\mathrm{V}}$ \\
\hline \multirow[t]{3}{*}{ Histidine } & \multirow{3}{*}{$\begin{array}{l}7.79\left(\mathrm{~d},{ }^{2} \mathrm{CH}\right), 7.06\left(\mathrm{~d},{ }^{5} \mathrm{CH}\right), \\
3.98\left(\mathrm{dd},{ }^{\alpha} \mathrm{CH}\right), 3.12(\mathrm{dd}, \\
\left.{ }^{\beta} \mathrm{CH}_{2}\right)\end{array}$} & Brain & $0.57-1.0$ & O. mykiss $\mathrm{A}^{\mathrm{A}}$ \\
\hline & & Muscle & $0.21-0.56 / \sim 0.55$ & P. lethostigma ${ }^{\mathrm{B}} / O$. mordax $^{\mathrm{G}}$ \\
\hline & & Plasma & $0.14-0.21$ & O. mykiss ${ }^{\mathrm{A}}$ \\
\hline \multirow[t]{3}{*}{ Tryptophan } & \multirow{3}{*}{$\begin{array}{c}7.72\left(\mathrm{~d},{ }^{4} \mathrm{CH}\right), 7.53\left(\mathrm{~d},{ }^{7} \mathrm{CH}\right), \\
7.31\left(\mathrm{~s},{ }^{2} \mathrm{CH}\right), 7.28\left(\mathrm{t},{ }^{5} \mathrm{CH}\right), \\
4.05\left(\mathrm{dd},{ }^{\alpha} \mathrm{CH}\right), 3.47(\mathrm{dd}, \\
\left.{ }^{\beta} \mathrm{CH}_{2}\right), 3.29\left(\mathrm{dd},{ }^{\beta} \mathrm{CH}_{2}\right)\end{array}$} & Brain & $\sim 0.06$ & O. mykiss ${ }^{\mathrm{A}}$ \\
\hline & & Muscle & $0.36-0.39$ & O. $\operatorname{mordax}^{\mathrm{G}}$ \\
\hline & & Plasma & $\sim 0.04$ & O. mykiss ${ }^{\mathrm{A}}$ \\
\hline \multirow[t]{3}{*}{ Lysine } & \multirow{3}{*}{$\begin{array}{c}3.74\left(\mathrm{t},{ }^{2} \mathrm{CH}\right), 3.02\left(\mathrm{t},{ }^{6} \mathrm{CH}_{2}\right), \\
1.89\left(\mathrm{~m},{ }^{3} \mathrm{CH}_{2}\right), 1.71(\mathrm{~m}, \\
\left.{ }^{5} \mathrm{CH}_{2}\right), 1.46\left(\mathrm{~m},{ }^{4} \mathrm{CH}_{2}\right)\end{array}$} & Brain & $0.12-0.33$ & O. mykiss ${ }^{\mathrm{A}}$ \\
\hline & & Muscle & $1.01-1.18 / 0.16-0.20$ & P. lethostigma ${ }^{\mathrm{B}} / O$. mordax $^{\mathrm{G}}$ \\
\hline & & Plasma & $0.08-0.47$ & O. mykiss ${ }^{\mathrm{A}}$ \\
\hline \multirow[t]{3}{*}{ Arginine } & \multirow{3}{*}{$\begin{array}{l}3.76\left(\mathrm{t},{ }^{2} \mathrm{CH}\right), 3.23\left(\mathrm{t},{ }^{5} \mathrm{CH}_{2}\right), \\
1.90\left(\mathrm{~m},{ }^{3} \mathrm{CH}_{2}\right), 1.68(\mathrm{~m}, \\
\left.{ }^{4} \mathrm{CH}_{2}\right)\end{array}$} & Brain & $0.11-0.15$ & O. mykiss ${ }^{\mathrm{A}}$ \\
\hline & & Muscle & $0.09-0.38 / \sim 0.05$ & P. lethostigma ${ }^{\mathrm{B}} / O$. mordax $^{\mathrm{G}}$ \\
\hline & & Plasma & $0.08-0.22$ & O. mykiss ${ }^{\mathrm{A}}$ \\
\hline \multirow[t]{2}{*}{ Cysteine } & \multirow{2}{*}{$\begin{array}{l}3.97\left(\mathrm{dd},{ }^{2} \mathrm{CH}\right), 3.06(\mathrm{~m}, \\
\left.{ }^{3} \mathrm{CH}_{2}\right)\end{array}$} & Brain & ND & O. mykiss ${ }^{\mathrm{A}}$ \\
\hline & & Plasma & $<0.01$ & O. mykiss ${ }^{\mathrm{A}}$ \\
\hline \multirow[t]{3}{*}{ Taurine } & \multirow[t]{3}{*}{$3.41\left(\mathrm{t},{ }^{1} \mathrm{CH}_{2}\right), 3.25\left(\mathrm{t},{ }^{2} \mathrm{CH}_{2}\right)$} & Brain & $17.5-19.3 / 17.2 / 4.59$ & $\begin{array}{l}\text { O. mykiss } \mathrm{A}^{\mathrm{A}} / O . \text { mykiss }^{\mathrm{D}} / D . \\
\text { rario }^{\mathrm{I}}\end{array}$ \\
\hline & & Muscle & $16.5-41.3 / 10.1-12.6$ & P. lethostigma ${ }^{\mathrm{B}} / O$. mordax $^{\mathrm{G}}$ \\
\hline & & Plasma & $0.23-0.51 / 0.77-0.92$ & O. mykiss ${ }^{\mathrm{A}} / S$. salar $\mathrm{V}^{\mathrm{V}}$ \\
\hline \multirow[t]{3}{*}{ Glutathione (reduced) } & \multirow{3}{*}{$\begin{array}{l}4.53\left(\mathrm{dd},{ }^{7} \mathrm{CH}\right), 3.78(\mathrm{~s}, \\
\left.{ }^{10} \mathrm{CH}_{2}\right), 2,97\left(\mathrm{dd},{ }^{7} \mathrm{CH}_{2}\right), \\
2,54\left(\mathrm{~m},{ }^{4} \mathrm{CH}_{2}\right), 2.13(\mathrm{~m}, \\
\left.{ }^{3} \mathrm{CH}_{2}\right)\end{array}$} & Brain & 1.18 & D. rario $^{\mathrm{I}}$ \\
\hline & & Liver & $1.08-1.57$ & S. alpinus ${ }^{\mathrm{F}}$ \\
\hline & & Blood & $0.36-0.49$ & S. alpinus $^{\mathrm{F}}$ \\
\hline \multirow[t]{2}{*}{ GABA } & \multirow{2}{*}{$\begin{array}{l}3.01\left(\mathrm{~m},{ }^{2} \mathrm{CH}_{2}\right), 2.28(\mathrm{t}, \\
\left.{ }^{4} \mathrm{CH}_{2}\right), 1.89\left(\mathrm{qu},{ }^{3} \mathrm{CH}_{2}\right)\end{array}$} & Brain & $3.05 / 0.89$ & O. mykiss ${ }^{\mathrm{D}} /$ D. rario $^{\mathrm{I}}$ \\
\hline & & Retina & $1.65-3.15$ & C. auratus $^{\mathrm{E}}$ \\
\hline NAA & $\begin{array}{l}7.82(\mathrm{~d}, \mathrm{NH}), 4.38\left(\mathrm{dd},{ }^{2} \mathrm{CH}\right), \\
2.67\left(\mathrm{dd},{ }^{3} \mathrm{CH}_{2}\right), 2.49(\mathrm{dd}, \\
\left.{ }^{3} \mathrm{CH}_{2}\right), 2.01\left(\mathrm{~s},{ }^{2} \mathrm{CH}_{3}\right)\end{array}$ & Brain & 5.43 & D. rario $^{\mathrm{I}}$ \\
\hline Carnitine (total) & $\begin{array}{l}4.55\left(\mathrm{~m},{ }^{3} \mathrm{CH}\right), 3.42(\mathrm{~m}, \\
\left.{ }^{4} \mathrm{CH}_{2}\right) 3.23\left[\mathrm{~s}, \mathrm{~N}\left(\mathrm{CH}_{3}\right)_{3}\right] \\
2.45\left(\mathrm{dd},{ }^{2} \mathrm{CH}_{2}\right)\end{array}$ & Muscle & $212-367$ & S. acanthias ${ }^{\mathrm{H}}$ \\
\hline \multicolumn{5}{|l|}{ Organic acids } \\
\hline \multirow[t]{2}{*}{ Formate } & \multirow[t]{2}{*}{$8.44\left(\mathrm{~s},{ }^{1} \mathrm{CH}\right)$} & Muscle & $0.54-0.65 / 0.86-2.48$ & Tuna \& Mackerel ${ }^{\mathrm{J}} /$ Mackerel $^{\mathrm{K}}$ \\
\hline & & Muscle (spoiled) & $4.3-7.8$ & Tuna \& Mackerel ${ }^{\mathrm{J}}$ \\
\hline \multirow[t]{2}{*}{ Acetate } & \multirow[t]{2}{*}{$1.90\left(\mathrm{~s},{ }^{2} \mathrm{CH}_{3}\right)$} & Muscle & $0.33-0.81 / 0.48-5.10$ & Tuna \& Mackerel $^{\mathrm{J}} /$ Mackerel $^{\mathrm{K}}$ \\
\hline & & Muscle (spoiled) & Up to $\sim 5$ & 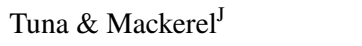 \\
\hline Lactate & $4.12\left(\mathrm{q},{ }^{2} \mathrm{CH}\right), 1.31\left(\mathrm{~d},{ }^{3} \mathrm{CH}_{3}\right)$ & Brain & 3.70 & D. rario $^{\mathrm{I}}$ \\
\hline & & Muscle & 3.0 & A. ocellatus $\mathrm{Q}$ \\
\hline & & Plasma & $2.31-7.44$ & B. cephalus $^{\mathrm{R}}$ \\
\hline Succinate & $2.41\left(\mathrm{~s},{ }^{2} \mathrm{CH}_{2},{ }^{3} \mathrm{CH}_{2}\right)$ & Mantle & Up to 15 & L. elliptica ${ }^{\mathrm{L}}$ \\
\hline & & Adductor muscle & $0.19-3.30$ & A. pectinata japonica ${ }^{\mathrm{M}}$ \\
\hline Fumarate & $6.51\left(\mathrm{~s},{ }^{2} \mathrm{CH},{ }^{3} \mathrm{CH}\right)$ & Adductor muscle & $0.20-1.01$ & A. pectinata japonica ${ }^{\mathrm{M}}$ \\
\hline Malonate & $3.11\left(\mathrm{~s},{ }^{2} \mathrm{CH}_{2}\right)$ & - & - & - \\
\hline$\beta$-hydroxybutyrate & $\begin{array}{l}4.16\left(\mathrm{~m},{ }^{3} \mathrm{CH}\right), 2.41(\mathrm{~m}, \\
\left.{ }^{2} \mathrm{CH}_{2}\right), 2.31\left(\mathrm{~m},{ }^{2} \mathrm{CH}_{2}\right), 1.02 \\
\left(\mathrm{~d},{ }^{4} \mathrm{CH}_{3}\right)\end{array}$ & Muscle & $0.99-2.00$ & S. acanthias $^{\mathrm{H}}$ \\
\hline
\end{tabular}


Table 3 (continued)

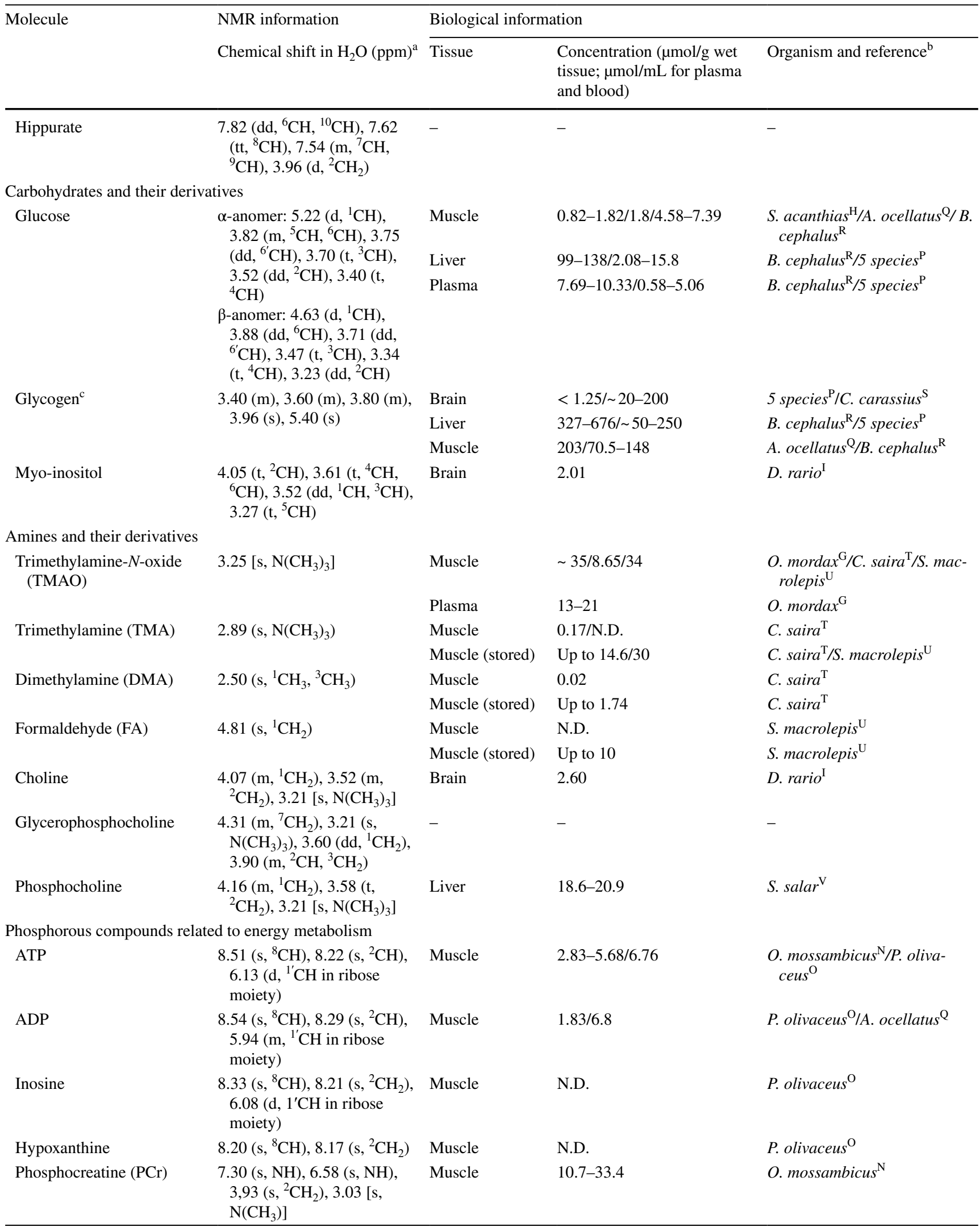


Table 3 (continued)

\begin{tabular}{|c|c|c|c|c|}
\hline \multirow[t]{2}{*}{ Molecule } & \multirow{2}{*}{$\begin{array}{l}\text { NMR information } \\
\text { Chemical shift in } \mathrm{H}_{2} \mathrm{O}(\mathrm{ppm})^{\mathrm{a}}\end{array}$} & \multicolumn{3}{|c|}{ Biological information } \\
\hline & & Tissue & $\begin{array}{l}\text { Concentration ( } \mu \mathrm{mol} / \mathrm{g} \text { wet } \\
\text { tissue; } \mu \mathrm{mol} / \mathrm{mL} \text { for plasma } \\
\text { and blood) }\end{array}$ & Organism and reference ${ }^{b}$ \\
\hline Creatine & $\begin{array}{c}6.65(\mathrm{~s}, \mathrm{NH}), 3,91\left(\mathrm{~s},{ }^{2} \mathrm{CH}_{2}\right), \\
3.03\left[\mathrm{~s}, \mathrm{~N}\left(\mathrm{CH}_{3}\right)\right]\end{array}$ & Muscle & 11.6 & A. ocellatus ${ }^{\mathrm{Q}}$ \\
\hline
\end{tabular}

ND Not detected

${ }^{a}$ Only chemical shifts of major peaks are shown for convenience. 4,4-dimethyl-4-silapentane-1-sulfonic acid-trimethyl singlet resonance was used as the reference. Data cited from: Brandao et al. (2015), Cappello et al. (2016), Ekman et al. (2007), and Govindaraju et al. (2000), and the Human Metabolome Database (Wishart et al. 2008)

${ }^{\mathrm{b}}$ Scientific names: Atrina pectinata japonica, Astronotus ocellatus, Brycon cephalus, Carassius carassius, Cololabis saira, Laternula elliptica, Oreochromis mossambicus, Paralichthys lethostigma, Paralichthys olivaceus, Salmo salar, Salvelinus alpinus, Saurida macrolepis, Squalus acanthias

${ }^{\mathrm{c}}$ Refer to Zang et al. 1991 for peak assignment. Concentration is represented by glycosyl units (micromols per gram wet tissue)

References: ${ }^{\mathrm{A}}$ Yamamoto et al. (2000), ${ }^{\mathrm{B}}$ Lasserre and Gilles (1971), ${ }^{\mathrm{C}}$ Wilkie et al. (2015), ${ }^{\mathrm{D}}$ Seiler et al. (1973), ${ }^{\mathrm{E}}$ Lam (1972), ${ }^{\mathrm{F}}$ Ritola et al. (2000), ${ }^{\mathrm{G}}$ Treberg et al. (2005), ${ }^{\mathrm{H}}$ Richards et al. (2003), ${ }^{\mathrm{I}}$ Kabli et al. (2009), ${ }^{\mathrm{J}}$ Miyahara (1961) (scientific name not available), ${ }^{\mathrm{K}}$ Moriyama et al. (1964) (scientific name not available), ${ }^{\mathrm{L}}$ Peck et al. (2002), ${ }^{\mathrm{M}}$ Inoue et al. (2007), ${ }^{\mathrm{N}}$ van Ginneken et al. (1999), ${ }^{\mathrm{O}}$ Ogata et al. (2018), ${ }^{\mathrm{P} S h o r t}$ et al. (2018), ${ }^{\mathrm{Q}}$ Richards et al. (2007), ${ }^{\mathrm{R}}$ Vieira et al. (2005), ${ }^{\mathrm{S}}$ Vornanen et al. (2006), ${ }^{\mathrm{T}}$ Sato et al. (2011), ${ }^{\mathrm{U}}$ Fukushima (2016), ${ }^{\mathrm{V}}$ Espe et al. (2010)

derived from biochemical measurements. For tissue samples, amounts of metabolites (micromols per gram tissue or milligrams per $100 \mathrm{~g}$ tissue) are more informative data that can be derived from NMR spectra, as seen in several previous studies (van Ginneken et al. 1996, 1999). On the other hand, lipophilic metabolites such as fatty acids are difficult to identify using ${ }^{1} \mathrm{H}$ NMR spectroscopy because they usually have broad and overlapping peaks. ${ }^{1} \mathrm{H}$ NMR spectroscopy can determine the global unsaturation level and the ratio of saturated, monounsaturated, and polyunsaturated fatty acids, even in vivo (Branca and Warren 2011), but gas chromatography is a more reliable method for the identification of individual fatty acids in biological samples.

Below we highlight ${ }^{1} \mathrm{H}$ NMR metabolomics studies on aquatic organisms with respect to research interests. Interested readers are directed to several specialized reviews on NMR metabolomics using aquatic organisms (Alfaro and Young 2018; Samuelsson and Larsson 2008; Young and Alfaro 2018).

Starvation dramatically affects fish metabolism, and ${ }^{1} \mathrm{H}$ NMR has been used to understand the integrated metabolic status of fish under starvation (Kokushi et al. 2011; Kullgren et al. 2010; Segner et al. 1997). Typical responses of fish to starvation include: (1) glycogen degradation, (2) lipid mobilization, and (3) protein degradation followed by hepatic gluconeogenesis, as confirmed in many species (Kaneko et al. 2016b; Khieokhajonkhet et al. 2016; Kullgren et al. 2010; Sheridan 1994). In general these processes take place in the above order, but the actual time course depends on many factors. Metabolic transition can be best illustrated by monitoring metabolite levels in plasma and other tissues. For example, ${ }^{1} \mathrm{H}$ NMR metabolomics detected the increase in very low density lipoprotein in plasma with a concomitant increase in plasma alanine levels in rainbow trout Oncorhynchus mykiss starved for 28 days, indicating that muscle protein catabolism takes place in parallel with lipid mobilization (Kullgren et al. 2010). Common carp Cyprinus carpio starved for 14 days had increased plasma ketone levels, but not amino acid levels, suggesting that lipids are the main energy source under this condition (Kokushi et al. 2011). Importantly, alanine levels often change under starvation, which is consistent with a previous ${ }^{14} \mathrm{C}$ labeling study that showed that alanine is the most effectively utilized amino acid in the liver of rainbow trout (French et al. 1981). Alanine levels are also frequently affected by physical and chemical stresses (Brandao et al. 2015; Ekman et al. 2007; Viant et al. 2003), although both increase and decrease have been reported, possibly reflecting the complex interactions between organs. In addition to starvation, the effects of various diets on fish metabolism have been actively investigated by ${ }^{1} \mathrm{H}$ NMR metabolomics (Casu et al. 2017; Shen et al. 2017; Wei et al. 2017).

Exposure to toxic contaminants is also a major challenge for organisms living in aquatic environments. ${ }^{1} \mathrm{H}$ NMR metabolomics has been conducted using fish and aquatic invertebrates exposed to heavy metals such as mercury (Brandao et al. 2015; Cappello et al. 2016), cadmium (Zhang et al. 2011), and copper (Santos et al. 2010). Samples included both laboratory-exposed organisms and wild ones caught in contaminated areas. Heavy metal exposure has been associated with low levels of reduced-form glutathione (Cappello et al. 2016) or high levels of total glutathione (Brandao et al. 2015), suggesting that oxidative stress is a 
key mechanism of heavy metal toxicity. High phosphocholine levels have been reported in fish captured from a highly contaminated area (Brandao et al. 2015). Phosphocholine is a degradation product of phosphatidylcholine (PC), a major membrane phospholipid, and is therefore indicative of membrane damage or active membrane turnover caused by heavy metals. In addition to heavy metals, ${ }^{1} \mathrm{H}$ NMR metabolomics has also been used to investigate effects of endocrine disruptors (Ekman et al. 2008, 2009; Katsiadaki et al. 2010; Samuelsson et al. 2006), which are known to have fundamental effects on reproduction of aquatic organisms (Porseryd et al. 2018; Yoon et al. 2008). Endocrine disruptors had sex-dependent effects on the hepatic content of PC, possibly affecting various membrane-dependent processes such as lipoprotein formation, vitellogenin synthesis, and bile formation (Ekman et al. 2009). ${ }^{1} \mathrm{H}$ NMR metabolomics have also been used to assess the effect of other environmental toxicants such as oil (Kokushi et al. 2012), polycyclic aromatic hydrocarbons (Cappello et al. 2013), pesticides/ herbicides (Viant et al. 2006a, b; Wang et al. 2017a), and contaminated sediments (Williams et al. 2014).

Other studies have investigated the effect of physical stresses using ${ }^{1} \mathrm{H}$ NMR metabolomics. There are a number of studies on the effect of thermal stress on the metabolism of fish (Kullgren et al. 2013; Viant et al. 2003) and aquatic invertebrates (Lannig et al. 2010; Shao et al. 2015). Again, choline metabolism is frequently affected by temperature change, possibly reflecting the reorganization of plasma membranes. Temperature has been found to affect amino acid metabolism in almost all studies, but it has been difficult for researchers to provide an integrative discussion about this since temperature can regulate amino acid metabolism both positively and negatively. In this context, NMR metabolomics would be more useful in complementing other omics data in a single study, rather than providing the means for comparability across studies, especially when examining complex phenomena like temperature change. One interesting finding from ${ }^{1} \mathrm{H}$ NMR metabolomics is that low temperature possibly affects metabolism via the cortisol and insulin pathways (Melis et al. 2017), which is similar to the mechanism whereby handling stress affects metabolism (Karakach et al. 2009). ${ }^{1} \mathrm{H}$ NMR metabolomics is a promising technology for investigating the function of these hormones using cultured cells or tissues of aquatic organisms.

\section{In vivo NMR spectroscopy and magnetic resonance spectroscopy}

\section{Principle and characteristics}

NMR spectroscopy is a non-invasive technique because electromagnetic waves at the resonance frequency have low energy. This has led to the development of two major in vivo applications of NMR. One is in vivo NMR spectroscopy or magnetic resonance spectroscopy (MRS) that acquires NMR signals, similar to those shown in Fig. 2, directly from specimens. The other is in vivo NMR imaging or MRI, which will be described later. These two technologies are used concomitantly. Prior to in vivo NMR spectroscopy, specimens are placed in an MRI scanner to acquire three-dimensional MRI data. Then, a voxel is placed in the three-dimensional MRI space to encompass the area from which in vivo NMR spectroscopy signals will be acquired. The in vivo NMR spectroscopy thus reveals the total amount of metabolites within the voxel. A strong and homogeneous external magnetic field facilitates highly sensitive acquisition and the use of small voxels (i.e., high temporal and spatial resolution).

In vivo ${ }^{31} \mathrm{P}$ NMR spectroscopy achieved earlier technical success than other in vivo NMR approaches because of its relatively high sensitivity and limited number of target metabolites (Table 2). In vivo ${ }^{31} \mathrm{P}$ NMR is capable of illustrating energy state changes by quantifying PCr, ATPrelated compounds, and $\mathrm{Pi}$ with a temporal resolution of a few seconds (Liu et al. 2017). As mentioned above, these metabolites undergo rapid post-mortem changes, and therefore in vivo monitoring is necessary to accurately trace their dynamics. Voxels of about $500 \mathrm{~mm}^{3}$ are commonly used in human studies, while in small animal studies the voxels can be as small as $3.6 \mathrm{~mm}^{3}$ (Liu et al. 2017).

In vivo ${ }^{13} \mathrm{C}$ NMR spectroscopy has been used to detect various metabolites. However, due to its low sensitivity, natural abundance in vivo ${ }^{13} \mathrm{C}$ NMR spectroscopy is most suitable for the quantification of relatively abundant metabolites such as glycogen and lipids. The low natural abundance of ${ }^{13} \mathrm{C}$, on the other hand, facilitates the use of in vivo ${ }^{13} \mathrm{C}$ NMR spectroscopy for tracing ${ }^{13} \mathrm{C}$-labeled metabolites. This application has been highly fruitful in mammals, as reviewed in the section of stable isotope tracing by NMR.

Compared to in vivo ${ }^{31} \mathrm{P}$ and in vivo ${ }^{13} \mathrm{C}$ NMR, the in vivo application of ${ }^{1} \mathrm{H}$ NMR has several difficulties (de Graaf 2007). Firstly, the water proton signal is up to several orders of magnitude higher than signals from metabolites of interest, requiring a water-suppression process. In liquid ${ }^{1} \mathrm{H}$ NMR spectroscopy, deuterium oxide can be used as the aqueous solvent to reduce the water proton signal. Secondly, macromolecules including protein and lipids cause broad peaks that overlap those of the metabolites of interest. In liquid ${ }^{1} \mathrm{H}$ NMR spectroscopy, such macromolecules can be removed during the extraction process. Thirdly, ${ }^{1} \mathrm{H}$ NMR spectroscopy gives many peaks within a narrow chemical shift range compared to other NMR applications. Accordingly, only up to 20 different metabolites of mammalian tissues can be quantified by state-of-the-art in vivo ${ }^{1} \mathrm{H}$ NMR spectroscopy (de Graaf 2007). However, its high sensitivity 
enables in vivo quantification of metabolites at relatively low concentrations ( 0.5 mM) (de Graaf 2005).

\section{In vivo NMR spectroscopy and MRS studies in aquatic biology}

Gold fish, crucian carp, and bitterling have extreme resistance to anoxia, whereas common carp does not despite its close phylogenetic relationship to these species. In general, factors related to resistance to anoxia include: (1) amount of glycogen, (2) end-product inhibition of glycolysis, (3) ability to stabilize intracellular $\mathrm{pH}$, and (4) ability to suppress metabolism. All of the relevant parameters can be readily evaluated by in vivo ${ }^{31} \mathrm{P}$ NMR spectroscopy. An outstanding series of in vivo ${ }^{31} \mathrm{P}$ NMR spectroscopy studies has demonstrated the mechanisms responsible for anoxia tolerance in the above species (van den Thillart and van Waarde 1996).

An early in vivo ${ }^{31} \mathrm{P}$ NMR study on fish anaerobiosis demonstrated that goldfish have high hypoxia/anoxia tolerance compared to common carp because of metabolic suppression and a capability to switch the glycolytic endproduct from lactate to ethanol (van den Thillart et al. 1989). van den Thillart et al. (1989) measured the ATPrelated compounds and intracellular $\mathrm{pH}$ at 10-min intervals in the muscle of unanesthetized carp and goldfish before, during, and after a period of anoxia. In carp muscle, anoxia caused a continuous decline in $\mathrm{PCr}$ and intracellular $\mathrm{pH}$. In contrast, in goldfish a decrease of $\mathrm{PCr}$ and intracellular $\mathrm{pH}$ under anoxia was prevented by the suppression of metabolism and synthesis of ethanol, not lactate, as the glycolytic end-product. Ethanol can be excreted into the surrounding water, and therefore does not exert a significant effect on fish muscle metabolism. Furthermore, in vivo ${ }^{31} \mathrm{P}$ NMR spectroscopy has been applied to swimming fish demonstrating that anaerobic metabolism takes place at speeds equal to and greater than $70 \%$ of the critical swimming speed in rainbow trout (Burgetz et al. 1998). Detailed in vivo metabolic profiling also revealed that the $\mathrm{PCr} / \mathrm{Cr}$ ratio is higher than previously thought [more than $80 \%$ by in vivo measurement (van Waarde et al. 1990)]; a maximum value of $45 \%$ had been previously reported by post-mortem determination (Dobson and Hochachka 1987)]. Several in vivo ${ }^{31}$ P NMR spectroscopy studies have also been carried out from ecotoxicological (Viant et al. 2006a, b) and physiological (Martello et al. 1998; Maus et al. 2018) perspectives.

In contrast, there are a limited number of in vivo ${ }^{1} \mathrm{H}$ and ${ }^{13} \mathrm{C}$ NMR spectroscopy studies on aquatic organisms. Effects of hypoxia and season on metabolism have been addressed in a lugworm Arenicola marina by the combination of in vivo ${ }^{31} \mathrm{P}$ and ${ }^{13} \mathrm{C}$ NMR spectroscopy (Juretschke and Kamp 1995; Kamp et al. 1995), and in vivo ${ }^{1} \mathrm{H}$ NMR spectroscopy has been applied to non-anaesthetized marine fish (Bock et al.

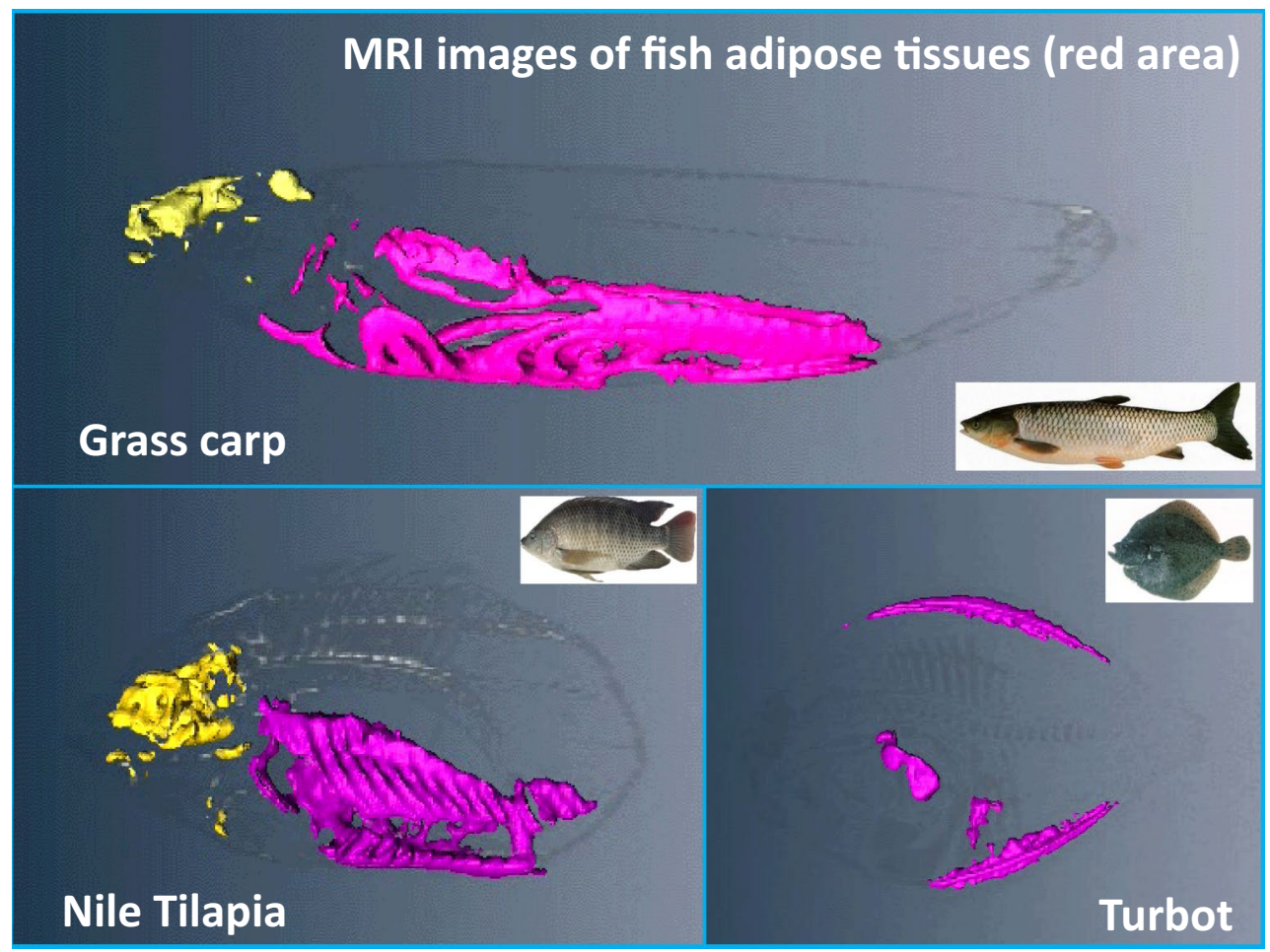

Fig. 3 Examples of three-dimensional magnetic resonance images of fish illustrating the distribution of adipose tissue. Mesenteric adipose tissue is shown in red, whereas other high T1 signal regions are shown in yellow. See Wu et al. (2015) for details 
2002). However, only a few metabolites, including lactate, choline, creatine and glycine, have been successfully quantified in these studies. A future challenge is to establish high resolution in vivo ${ }^{1} \mathrm{H}$ and ${ }^{13} \mathrm{C}$ NMR spectroscopy systems optimized for aquatic organisms. Muscle tissue, eggs, and whole fish bodies were subjected to in vivo ${ }^{1} \mathrm{H}$ NMR spectroscopy in recent studies to test the feasibility of novel pulse sequences (Cai et al. 2014, 2016).

\section{Stable isotope tracing by NMR}

\section{Principle and characteristics}

When exploring metabolic pathways in a given biological system, tracing of labeled metabolite(s) is essential. Traditionally ${ }^{3} \mathrm{H},{ }^{14} \mathrm{C}$, and ${ }^{32} \mathrm{P}$ have been used as tracers, but utilization of these radioactive nuclei requires special equipment. Furthermore, it is not very informative to follow these radioactive labels after substrates have been metabolized. For example, radioactive signals of ${ }^{14} \mathrm{C}$-glucose are indistinguishable from those of ${ }^{14} \mathrm{C}$-lactate, a metabolized product of ${ }^{14} \mathrm{C}$-glucose, unless these molecules can be identified by other techniques.

In vivo ${ }^{13} \mathrm{C}$ NMR spectroscopy following the infusion of ${ }^{13} \mathrm{C}$-labeled substrates overcomes these problems and provides an excellent means of exploring metabolic pathways. ${ }^{13} \mathrm{C}$ is a stable isotope with no radioactivity, and its low natural abundance results in high signal-to-noise ratios (Table 2). Moreover, NMR spectroscopy is able to distinguish labeled metabolites from chemical shift values-in the above example, ${ }^{13} \mathrm{C}$-glucose and ${ }^{13} \mathrm{C}$-lactate can be easily distinguished by chemical shift values in a single NMR acquisition. The tempting combination of ${ }^{13} \mathrm{C}$ labeling and in vivo ${ }^{13} \mathrm{C}$ NMR spectroscopy captured several important aspects of mammalian metabolism, such as the role of muscle glycogen synthesis in insulin resistance associated with type 2 diabetes (Shulman and Rothman 2005) and the strong stoichiometric coupling between glutamate neurotransmission and glucose oxidation in the brain, where more than two-thirds of the energy from glucose oxidation is used for glutamate neurotransmission and associated events (Rothman et al. 2003).

Proton-observed carbon-edited (POCE) NMR is an indirect detection method for ${ }^{13} \mathrm{C}$, which specifically and quantitatively discriminates protons attached to ${ }^{13} \mathrm{C}$ from those attached to ${ }^{12} \mathrm{C}$ (de Graaf et al. 2003, 2011). Namely, POCE NMR acquires two proton spectra. The total spectrum is the same as the normal ${ }^{1} \mathrm{H}$ NMR spectrum, while the inverted spectrum is edited for carbon-signals from protons bound to ${ }^{13} \mathrm{C}$ are selectively inverted. By subtracting these spectra, signals from ${ }^{13} \mathrm{C}$-binding protons can be specifically obtained. The ${ }^{1} \mathrm{H}$ acquisition in POCE NMR improved sensitivity up to 11 times for quantification of lactate compared to ${ }^{13} \mathrm{C}$ acquisition, encouraging the use of

${ }^{13} \mathrm{C}$ labeling (Rothman et al. 1985). There have been several successful applications of in vivo POCE NMR to mammals (Kaneko et al. 2017; van Eijsden et al. 2010), but to our knowledge this method has never been applied to non-mammalian models.

The most significant feature of these metabolic tracing technologies is that the high time resolution measurement of ${ }^{13} \mathrm{C}$-labeled metabolites enables the determination of metabolic flux of a given pathway, which is defined as the number of molecules flowing through the metabolic pathway per unit time (typically micromols per minute per gram tissue). The metabolic flux is an informative parameter for a given metabolic pathway that desirably should be investigated together with enzymatic activity, protein levels, and transcript levels. The relationship between enzymatic activity and metabolic flux is not linear, but often hyperbolic, and metabolic flux is likely to be more associated with phenotype than enzymatic activity (Fell 1992; Wang and Dykhuizen 2001). Deuterium $\left({ }^{2} \mathrm{H}\right)$ labeling has been used in several studies, but ${ }^{2} \mathrm{H}$ NMR spectroscopy has very low sensitivity (Table 2 ).

\section{Metabolic tracing studies in aquatic biology}

An important hypothesis that can be addressed by metabolic tracing is that lipid and amino acids, but not carbohydrate, are the preferred energy sources of fish. This hypothesis has long been acknowledged based on nutritional requirements and various biochemical parameters of fish (Hemre et al. 2002; Watanabe 1982; Wilson 1994). Other evidence supporting this hypothesis includes the high sensitivity of fish taste receptors to amino acids (Caprio 1975; Oike et al. 2007) and the low binding affinity of insulin-responsive glucose transporter to glucose (Capilla et al. 2004). However, although the effective utilization of lipids and amino acids compared to glucose has been sporadically reported by using ${ }^{14} \mathrm{C}$ labeling (Henderson and Sargent 1981; Shikata and Shimeno 1997), to our knowledge, there is no comprehensive and systematic study that has explored metabolic fluxes of these nutrients. Administration of ${ }^{13} \mathrm{C}$-labeled nutrients followed by time-course NMR quantification of the metabolized products would allow calculation of the metabolic flux of each nutrient into the TCA cycle, providing a novel link between molecular and nutritional/physiological aspects of fish metabolism.

Although not quantitative, it is important to note that several studies have utilized ${ }^{13} \mathrm{C}$ labeling to explore biosynthetic pathways of aquatic microorganisms (Murakami et al. 1998; Tsuda et al. 2001; Yamazaki et al. 2011). For example, Yamazaki et al. (2011) cultured a dinoflagellate Protoceratium reticulatum with several ${ }^{13} \mathrm{C}$-labeled substrates (e.g., acetate and glycolate), and detected the label flow by 
${ }^{13} \mathrm{C}$ NMR spectroscopy. The incorporation patterns of ${ }^{13} \mathrm{C}$ provided useful information about biosynthesis of a ladderframe polyether called yessotoxin. Increased sensitivity of NMR will lead to more comprehensive identification of metabolites synthesized from ${ }^{13} \mathrm{C}$ substrate, facilitating the discovery of novel metabolic pathways in aquatic organisms. Indeed, the first demonstration of ethanol synthesis in goldfish was also achieved by using ${ }^{14} \mathrm{C}$-lactate and ${ }^{14} \mathrm{C}$-glucose (Shoubridge and Hochachka 1980).

\section{MRI and related imaging technologies}

\section{Principle and characteristics}

In vivo NMR imaging, or MRI, is an NMR-based imaging technology that differs from in vivo NMR spectroscopy. The first important reports on MRI were those of Lauterbur (1973) and Mansfield and Grannell (1973), in which they reconstructed the spatial distribution of the spins of magnetic nuclei as images. In principle, signals from any magnetic nuclei can be presented in the form of an image. However, due to its abundance in biological systems, the signal of the water proton is used for most MRI applications. Lauterbur and Mansfield were awarded the 2003 Nobel Prize in Medicine for their discovery concerning MRI. Nowadays, various types of MRI images can be obtained by applying different pulse sequences.

The important clinical advantage of MRI is that it is a non-invasive method with relatively high resolution of $10 \mu \mathrm{m}$ to $1 \mathrm{~mm}$ (Hyder 2009). The use of MRI led to the development of several relevant imaging technologies. For example, diffusion tensor imaging is a modality of MRI that measures the directionality of diffusion of water molecules. Long and thin cells, such as neurons and muscle fibers, can be visualized by using this technology due to the directed diffusion of water molecules within these cells. Functional MRI (fMRI) is a technology used to map the physiological and metabolic processes of tissues, mainly the neuronal activation in the brain, based on blood oxygenation level measured in an MRI scanner (Ogawa et al. 1990). MRI for a simple imaging purpose is sometimes called structural or anatomical MRI in order to distinguish it from fMRI. Live specimens are required for fMRI, whereas ex vivo application has been very common in structural MRI.

\section{MRI studies in aquatic biology}

Structural MRI has been increasingly used in zoology because of its ability to depict soft tissues at high resolutions. Target species include metazoans from various taxa and even fossils (Ziegler et al. 2011). While many MRI studies in aquatic biology have been conducted from the viewpoint of food science using fish fillets (see Magnetic resonance technologies in seafood science), there have been several successful MRI applications from an anatomical perspective that visualized the internal structure of aquatic organisms such as bivalves (von Brand et al. 2009; Ziegler et al. 2011), sea urchins (Ziegler et al. 2008), and sea turtles (Valente et al. 2006). The Digital Fish Library containing MRI data for over 300 species is available from a public website (Berquist et al. 2012). Furthermore, the Digital Fish Library images are reconstructed into three-dimensional volumetric images and segmented as several structures, providing a great resource for comparative anatomy. The primary limiting factor for the more widespread use of MRI in zoology appears to be the size of specimens because MRI does not provide meaningful structural information from specimens less than $1 \mathrm{~mm}$ (Ziegler et al. 2011). However, a recent study successfully illustrated the physiological and metabolic differences between viscera and subcutaneous adipose tissues in Nile tilapia by taking advantage of structural MRI (Wang et al. 2017b). Further physiological applications will be forthcoming in the near future.

There are few fMRI studies on aquatic organisms. However, with appropriate experimental settings, it is possible to observe sensorimotor responses to temperature change (van den Burg et al. 2006, 2005) and olfactory responses to natal stream water (Bandoh et al. 2011) by using fMRI. The direct monitoring of brain activity by fMRI is also one of the future goals in aquatic biology field.

\section{Magnetic resonance technologies in seafood science}

Magnetic resonance technologies have been applied to seafood and post-mortem tissues of aquatic organisms (Erikson et al. 2012). The methodology used in this field is largely similar to the aforementioned NMR spectroscopy and MRI.

Trimethylamine- $\mathrm{N}$-oxide (TMAO) is an osmolyte found at relatively high concentrations in particular species such as walleye pollock Gadus chalcogrammus and sablefish Anoplopoma fimbria (Tokunaga 1970). During storage TMAO is reduced to trimethylamine (TMA), followed by decomposition to equimolar amounts of dimethylamine and formaldehyde (FA). Because TMA is responsible for the fishy smell, and FA cross-links muscle proteins resulting in poor texture, ${ }^{1} \mathrm{H}$ NMR detection of these substances is important for the assessment of seafood quality (Howell et al. 1996; Savorani et al. 2010).

Although the precise identification of fatty acids by NMR spectroscopy is difficult, as mentioned above, the content of omega-3 fatty acids, well-known components of seafood that are considered part of a healthy diet, can be quantified by NMR spectroscopy (Marcone et al. 2013). 
An interesting application of liquid NMR spectroscopy is that fatty acid peaks in NMR spectra can be used to classify wild and farmed fish by principle component analysis without identifying each fatty acid individually (Aursand et al. 2009; Rezzi et al. 2007). If in vivo application of this method can be developed in the future, it will be possible to non-invasively distinguish wild and farmed fish by lipid profiling.

Structural MRI is capable of distinguishing muscle from adipose tissue by applying certain pulse sequences (Fig. 3), and thus has been used to determine the fat content of fish (Collewet et al. 2013; Wu et al. 2015). It is especially important to use high-resolution imaging technology for this because there are many local fat depots (i.e., intramuscular adipose tissue) in fish muscle (Han et al. 2013; Kaneko et al. 2016a; Khieokhajonkhet et al. 2014). These studies ensured that MRI is a useful tool with which to determine seafood quality, especially when the number of samples is high. In addition to fat distribution, water and salt distribution have been determined by ${ }^{1} \mathrm{H}$ and ${ }^{23} \mathrm{Na}$ MRI, respectively (Aursand et al. 2008).

\section{Conclusion and future perspectives}

This review provides a broad, though not comprehensive, overview of magnetic resonance technologies used in aquatic biology and seafood science. Overall, the application of liquid NMR spectroscopy has been successful in the structural determination of natural compounds and metabolomics. Recent improvements in sensitivity and spectral resolution will further refine these applications, making NMR spectroscopy one of the indispensable technologies in the era of a multiomics approach. Application of MRI has also been fruitful in providing a means of non-invasive morphological assessment of aquatic organisms, especially in seafood science. Establishment of lower cost, multi-modal MRI systems (e.g., simultaneous acquisition of ${ }^{1} \mathrm{H}$ and ${ }^{23} \mathrm{Na}$ MRI to determine fat and salt contents) will further facilitate the use of MRI in these fields. On the other hand, there is plenty of room for future technological development of in vivo NMR spectroscopy and metabolic tracing. Although many major metabolic pathways of aquatic organisms have been identified at the genetic level (e.g., glycolysis and the TCA cycle), there must be considerable diversity in metabolic fluxes of these pathways associated with physiological diversity, as in the case of ethanol production in goldfish and the possible central role of lipids/amino acids in fish metabolism. Future exploration of such metabolic diversity should be valuable for further understanding and effective utilization of aquatic organisms.
Acknowledgements We are grateful to Dr. Zhen-Yu Du, East China Normal University, for providing fish MRI data. G. K. was supported by funding from the M. G. and Lillie A. Johnson Foundation, Victoria, Texas and from the Dean's Office at the University of Houston-Victoria.

Open Access This article is distributed under the terms of the Creative Commons Attribution 4.0 International License (http://creativeco mmons.org/licenses/by/4.0/), which permits unrestricted use, distribution, and reproduction in any medium, provided you give appropriate credit to the original author(s) and the source, provide a link to the Creative Commons license, and indicate if changes were made.

\section{References}

Alfaro AC, Young T (2018) Showcasing metabolomic applications in aquaculture: a review. Rev Aquac 10:135-152

Arnold JT, Dharmatti SS, Packard M (1951) Chemical effects on nuclear induction signals from organic compounds. J Chem Phys 19:507

Aursand IG, Veliyulin E, Böcker U, Ofstad R, Rustad T, Erikson U (2008) Water and salt distribution in Atlantic salmon (Salmo salar) studied by low-field ${ }^{1} \mathrm{H}$ NMR, ${ }^{1} \mathrm{H}$ and ${ }^{23} \mathrm{Na}$ MRI and light microscopy: effects of raw material quality and brine salting. $\mathrm{J}$ Agric Food Chem 57:46-54

Aursand M, Standal IB, Praël A, McEvoy L, Irvine J, Axelson DE (2009) ${ }^{13}$ C NMR pattern recognition techniques for the classification of Atlantic salmon (Salmo salar L.) according to their wild, farmed, and geographical origin. J Agric Food Chem 57:3444-3451

Bandoh H, Kida I, Ueda H (2011) Olfactory responses to natal stream water in sockeye salmon by BOLD fMRI. PLoS One 6:e16051

Beckonert O, Keun HC, Ebbels TM, Bundy J, Holmes E, Lindon JC, Nicholson JK (2007) Metabolic profiling, metabolomic and metabonomic procedures for NMR spectroscopy of urine, plasma, serum and tissue extracts. Nat Protoc 2:2692

Behar K, Den Hollander J, Stromski M, Ogino T, Shulman R, Petroff O, Prichard J (1983) High-resolution ${ }^{1} \mathrm{H}$ nuclear magnetic resonance study of cerebral hypoxia in vivo. Proc Natl Acad Sci USA 80:4945-4948

Berquist RM, Gledhill KM, Peterson MW, Doan AH, Baxter GT, Yopak KE, Kang N, Walker H, Hastings PA, Frank LR (2012) The digital fish library: using MRI to digitize, database, and document the morphological diversity of fish. PLoS One 7:e34499

Bloch F, Hansen WW, Packard M (1946) Nuclear induction. Phys Rev 69:127

Bock C, Sartoris F-J, Pörtner H-O (2002) In vivo MR spectroscopy and MR imaging on non-anaesthetized marine fish: techniques and first results. Magn Reson Imaging 20:165-172

Bollard ME, Stanley EG, Lindon JC, Nicholson JK, Holmes E (2005) NMR-based metabonomic approaches for evaluating physiological influences on biofluid composition. NMR Biomed 18:143-162

Branca RT, Warren WS (2011) In vivo NMR detection of diet-induced changes in adipose tissue composition. J Lipid Res 52:833-839

Brandao F, Cappello T, Raimundo J, Santos MA, Maisano M, Mauceri A, Pacheco M, Pereira P (2015) Unravelling the mechanisms of mercury hepatotoxicity in wild fish (Liza aurata) through a triad approach: bioaccumulation, metabolomic profiles and oxidative stress. Metallomics 7:1352-1363

Burgetz IJ, Rojas-Vargas A, Hinch SG, Randall DJ (1998) Initial recruitment of anaerobic metabolism during sub-maximal swimming in rainbow trout (Oncorhynchus mykiss). J Exp Biol 201:2711-2721 
Cai H, Chen Y, Cui X, Cai S, Chen Z (2014) High-resolution ${ }^{1} \mathrm{H}$ NMR spectroscopy of fish muscle, eggs and small whole fish via hadamard-encoded intermolecular multiple-quantum coherence. PLoS One 9:e86422

Cai H, Lin L, Ding S, Cui X, Chen Z (2016) Fast quantification of fatty acid profile of intact fish by intermolecular double-quantum coherence ${ }^{1} \mathrm{H}-\mathrm{NMR}$ spectroscopy. Eur J Lipid Sci Technol 118:1150-1159

Capilla E, Diaz M, Albalat A, Navarro I, Pessin JE, Keller K, Planas JV (2004) Functional characterization of an insulin-responsive glucose transporter (GLUT4) from fish adipose tissue. Am J Physiol Endocrinol Metab 287:E348-E357

Cappello T, Mauceri A, Corsaro C, Maisano M, Parrino V, Paro GL, Messina G, Fasulo S (2013) Impact of environmental pollution on caged mussels Mytilus galloprovincialis using NMR-based metabolomics. Mar Pollut Bull 77:132-139

Cappello T, Brandão F, Guilherme S, Santos MA, Maisano M, Mauceri A, Canário J, Pacheco M, Pereira P (2016) Insights into the mechanisms underlying mercury-induced oxidative stress in gills of wild fish (Liza aurata) combining ${ }^{1} \mathrm{H}$ NMR metabolomics and conventional biochemical assays. Sci Total Environ 548:13-24

Caprio J (1975) High sensitivity of catfish taste receptors to amino acids. Comp Biochem Physiol A Physiol 52:247-251

Casu F, Watson AM, Yost J, Leffler JW, Gaylord TG, Barrows FT, Sandifer PA, Denson MR, Bearden DW (2017) Metabolomics analysis of effects of commercial soy-based protein products in red drum (Sciaenops ocellatus). J Proteom Res 16:2481-2494

Collewet G, Bugeon J, Idier J, Quellec S, Quittet B, Cambert M, Haffray P (2013) Rapid quantification of muscle fat content and subcutaneous adipose tissue in fish using MRI. Food Chem 138:2008-2015

Cragg GM, Newman DJ (2001) Natural product drug discovery in the next millennium. Pharm Biol 39:8-17

de Graaf RA (2005) In vivo NMR spectroscopy-techniques; direct detection; MRS; kinetics and labels; fluxes; concentrations. In: Shulman R, Rothman DL (eds) Metabolomics by in vivo NMR. Wiley, Chichester, pp 7-29

de Graaf RA (2007) In vivo NMR spectroscopy. Wiley, West Sussex

de Graaf RA, Mason GF, Patel AB, Behar KL, Rothman DL (2003) In vivo ${ }^{1} \mathrm{H}-\left[{ }^{13} \mathrm{C}\right]-\mathrm{NMR}$ spectroscopy of cerebral metabolism. NMR Biomed 16:339-357

de Graaf RA, Chowdhury GM, Brown PB, Rothman DL, Behar KL (2009) In situ 3D magnetic resonance metabolic imaging of microwave-irradiated rodent brain: a new tool for metabolomics research. J Neurochem 109:494-501

de Graaf RA, Rothman DL, Behar KL (2011) State of the art direct ${ }^{13} \mathrm{C}$ and indirect ${ }^{1} \mathrm{H}-\left[{ }^{13} \mathrm{C}\right]$ NMR spectroscopy in vivo. A practical guide. NMR Biomed 24:958-972

Dobson G, Hochachka P (1987) Role of glycolysis in adenylate depletion and repletion during work and recovery in teleost white muscle. J Exp Biol 129:125-140

Ekman D, Teng Q, Jensen K, Martinovic D, Villeneuve D, Ankley G, Collette T (2007) NMR analysis of male fathead minnow urinary metabolites: a potential approach for studying impacts of chemical exposures. Aquat Toxicol 85:104-112

Ekman D, Teng Q, Villeneuve D, Kahl M, Jensen K, Durhan E, Ankley G, Collette T (2008) Investigating compensation and recovery of fathead minnow (Pimephales promelas) exposed to $17 \alpha$-ethynylestradiol with metabolite profiling. Environ Sci Technol 42:4188-4194

Ekman DR, Teng Q, Villeneuve DL, Kahl MD, Jensen KM, Durhan EJ, Ankley GT, Collette TW (2009) Profiling lipid metabolites yields unique information on sex- and time-dependent responses of fathead minnows (Pimephales promelas) exposed to $17 \alpha$-ethynylestradiol. Metabolomics 5:22
Erikson U, Standal IB, Aursand IG, Veliyulin E, Aursand M (2012) Use of NMR in fish processing optimization: a review of recent progress. Magn Reson Chem 50:471-480

Espe M, Rathore RM, Du ZY, Liaset B, El-Mowafi A (2010) Methionine limitation results in increased hepatic FAS activity, higher liver 18:1 to 18:0 fatty acid ratio and hepatic TAG accumulation in Atlantic salmon, Salmo salar. Amino Acids 39:449-460

Fell DA (1992) Metabolic control analysis: a survey of its theoretical and experimental development. Biochem J 286:313

Fenical W (1987) Marine soft corals of the genus Pseudopterogorgia: a resource for novel anti-inflammatory diterpenoids. J Nat Prod 50:1001-1008

French CJ, Mommsen TP, Hochachka PW (1981) Amino acid utilisation in isolated hepatocytes from rainbow trout. FEBS J $113: 311-317$

Fukushima H (2016) Effect of freezing on the control of formaldehyde formation. Nippon Suisan Gakkaishi 82:956 (In Japanese)

Govindaraju V, Young K, Maudsley AA (2000) Proton NMR chemical shifts and coupling constants for brain metabolites. NMR Biomed 13:129-153

Han Y, Kaneko G, Nagasaka R, Kondo H, Hirono I, Takahashi S-I, Watabe S, Ushio H (2013) Distribution of adipocyte-related cells in skeletal muscle of rainbow trout Oncorhynchus mykiss. Fish Sci 79:143-148

Hemre GI, Mommsen T, Krogdahl Å (2002) Carbohydrates in fish nutrition: effects on growth, glucose metabolism and hepatic enzymes. Aquacult Nutr 8:175-194

Henderson R, Sargent J (1981) Lipid biosynthesis in rainbow trout, Salmo gairdnerii, fed diets of differing lipid content. Comp Biochem Physiol C Comp Pharmacol 69:31-37

Howell N, Shavila Y, Grootveld M, Williams S (1996) High-resolution NMR and magnetic resonance imaging (MRI) studies on fresh and frozen cod (Gadus morhua) and haddock (Melanogrammus aeglefinus). J Sci Food Agric 72:49-56

Hyder F (2009) Dynamic imaging of brain function. In: Hyder F (ed) Dynamic brain imaging. Humana, New York, pp 3-21

Inoue S, Oshima Y, Abe SI, Wu RSS, Kai N, Honjo T (2007) Effects of tributyltin on the energy metabolism of pen shell (Atrina pectinata japonica). Chemosphere 66:1226-1229

Juretschke H, Kamp G (1995) In vivo nuclear magnetic resonance studies on the lugworm Arenicola marina. II. Seasonal changes of metabolism. J Comp Physiol B 165:153-160

Kabli S, Spaink HP, De Groot HJ, Alia A (2009) In vivo metabolite profile of adult zebrafish brain obtained by high-resolution localized magnetic resonance spectroscopy. J Magn Reson Imaging 29:275-281

Kamp G, Juretschke H-P, Thiel U, Englisch H (1995) In vivo nuclear magnetic resonance studies on the lugworm Arenicola marina. I. Free inorganic phosphate and free adenylmonophosphate concentrations in the body wall and their dependence on hypoxia. J Comp Physiol B 165:143-152

Kaneko G, Shirakami H, Hirano Y, Oba M, Yoshinaga H, Khieokhajonkhet A, Nagasaka R, Kondo H, Hirono I, Ushio H (2016a) Diversity of lipid distribution in fish skeletal muscle. Zool Sci 33:170-178

Kaneko G, Shirakami H, Yamada T, Ide SI, Haga Y, Satoh S, Ushio $\mathrm{H}$ (2016b) Short-term fasting increases skeletal muscle lipid content in association with enhanced mRNA levels of lipoprotein lipase 1 in lean juvenile red seabream (Pagrus major). Aquaculture 452:160-168

Kaneko G, Sanganahalli BG, Groman SM, Wang H, Coman D, Rao J, Herman P, Jiang L, Rich K, de Graaf RA, Taylor JR, Hyder F (2017) Hypofrontality and posterior hyperactivity in early schizophrenia: imaging and behavior in a preclinical model. Biol Psychiatry 81:503-513 
Karakach TK, Huenupi EC, Soo EC, Walter JA, Afonso LO (2009) ${ }^{1} \mathrm{H}-\mathrm{NMR}$ and mass spectrometric characterization of the metabolic response of juvenile Atlantic salmon (Salmo salar) to long-term handling stress. Metabolomics 5:123-137

Katsiadaki I, Williams TD, Ball JS, Bean TP, Sanders MB, Wu H, Santos EM, Brown MM, Baker P, Ortega F (2010) Hepatic transcriptomic and metabolomic responses in the stickleback (Gasterosteus aculeatus) exposed to ethinyl-estradiol. Aquat Toxicol 97:174-187

Keeler J (2010) Understanding NMR spectroscopy, 2nd edn. Wiley, New York

Kemp W (1986) NMR in chemistry-a multinuclear introduction. Macmillan, London

Khieokhajonkhet A, Kaneko G, Ohara K, Shirakami H, Ushio H (2014) Hormone-sensitive lipase in Japanese flounder Paralichthys olivaceus: the potential function of the inclinator muscle of fin as a lipid storage site. Fish Sci 80:341-352

Khieokhajonkhet A, Kaneko G, Hirano Y, Wang L, Ushio H (2016) Different effects of growth hormone and fasting on the induction patterns of two hormone-sensitive lipase genes in red seabream Pagrus major. Gen Comp Endocrinol 236:121-130

Kokushi E, Uno S, Koyama J (2011) Assessing the effects of starvation on freshwater carp Cyprinus carpio L. by ${ }^{1} \mathrm{H}-\mathrm{NMR}$ metabolomics approach. Nippon Suisan Gakkaishi 77:75-83 (In Japanese with English abstract)

Kokushi E, Uno S, Harada T, Koyama J (2012) ${ }^{1}$ H NMR-based metabolomics approach to assess toxicity of bunker a heavy oil to freshwater carp, Cyprinus carpio. Environ Toxicol 27:404-414

Kullgren A, Samuelsson LM, Larsson DJ, Björnsson BT, Bergman EJ (2010) A metabolomics approach to elucidate effects of food deprivation in juvenile rainbow trout (Oncorhynchus mykiss). Am J Physiol Regul Integr Comp Physiol 299:R1440-R1448

Kullgren A, Jutfelt F, Fontanillas R, Sundell K, Samuelsson L, Wiklander K, Kling P, Koppe W, Larsson DJ, Björnsson BT (2013) The impact of temperature on the metabolome and endocrine metabolic signals in Atlantic salmon (Salmo salar). Comp Biochem Physiol A Mol Integr Physiol 164:44-53

Kuznetsov G, Towle MJ, Cheng H, Kawamura T, TenDyke K, Liu D, Kishi Y, Melvin JY, Littlefield BA (2004) Induction of morphological and biochemical apoptosis following prolonged mitotic blockage by halichondrin B macrocyclic ketone analog E7389. Cancer Res 64:5760-5766

Lam DM (1972) The biosynthesis and content of gamma-aminobutyric acid in the goldfish retina. J Cell Biol 54:225-231

Lannig G, Eilers S, Pörtner HO, Sokolova IM, Bock C (2010) Impact of ocean acidification on energy metabolism of oyster, Crassostrea gigas - changes in metabolic pathways and thermal response. Mar Drugs 8:2318-2339

Lardon I, Nilsson GE, Stecyk JA, Vu TN, Laukens K, Dommisse R, De Boeck G (2013) ${ }^{1}$ H-NMR study of the metabolome of an exceptionally anoxia tolerant vertebrate, the crucian carp (Carassius carassius). Metabolomics 9:311-323

Lasserre P, Gilles R (1971) Modification of the amino acid pool in the parietal muscle of two euryhaline teleosts during osmotic adjustment. Experientia 27:1434-1435

Lauterbur P (1973) Image formation by induced local interactions: examples employing nuclear magnetic resonance. Nature 242:190-191

Le Belle J, Harris N, Williams S, Bhakoo K (2002) A comparison of cell and tissue extraction techniques using high-resolution ${ }^{1} \mathrm{H}$ NMR spectroscopy. NMR Biomed 15:37-44

Li K, Chung-Davidson Y-W, Bussy U, Li W (2015) Recent advances and applications of experimental technologies in marine natural product research. Mar Drugs 13:2694-2713
Lin CY, Wu H, Tjeerdema RS, Viant MR (2007) Evaluation of metabolite extraction strategies from tissue samples using NMR metabolomics. Metabolomics 3:55-67

Lira NS, Montes RC, Tavares JF, Silva MSd, Da Cunha EV, AthaydeFilho PFd, Rodrigues LC, Dias CdS, Barbosa-Filho JM (2011) Brominated compounds from marine sponges of the genus Aplysina and a compilation of their ${ }^{13} \mathrm{C}$ NMR spectral data. Mar Drugs 9:2316-2368

Liu Y, Gu Y, Yu X (2017) Assessing tissue metabolism by phosphorous-31 magnetic resonance spectroscopy and imaging: a methodology review. Quant Imaging Med Surg 7:707

Look SA, Fenical W, Matsumoto GK, Clardy J (1986) The pseudopterosins: a new class of antiinflammatory and analgesic diterpene pentosides from the marine sea whip Pseudopterogorgia elisabethae (Octocorallia). J Org Chem 51:5140-5145

Mansfield P, Grannell PK (1973) NMR 'diffraction' in solids? J Phys C: Solid State Physics 6:L422

Marcone MF, Wang S, Albabish W, Nie S, Somnarain D, Hill A (2013) Diverse food-based applications of nuclear magnetic resonance (NMR) technology. Food Res Int 51:729-747

Martello LB, Tjeerdema RS, Smith WS, Kauten RJ, Crosby DG (1998) Influence of salinity on the actions of pentachlorophenol in Haliotis as measured by in vivo ${ }^{31} \mathrm{P}$ NMR spectroscopy. Aquat Toxicol 41:229-250

Maus B, Bock C, Pörtner H-O (2018) Water bicarbonate modulates the response of the shore crab Carcinus maenas to ocean acidification. J Comp Physiol B 23:1-16

Melis R, Sanna R, Braca A, Bonaglini E, Cappuccinelli R, Slawski H, Roggio T, Uzzau S, Anedda R (2017) Molecular details on gilthead sea bream (Sparus aurata) sensitivity to low water temperatures from ${ }^{1} \mathrm{H}$ NMR metabolomics. Comp Biochem Physiol A Mol Integr Physiol 204:129-136

Miyahara S (1961) Gas-liquid chromatographic separation and determination of volatile fatty acids in fish meat during spoilage. Nippon Suisan Gakkaishi 27:42-47 (In Japanese with English abstract)

Molinski TF (2010) NMR of natural products at the 'nanomole-scale'. Nat Prod Rep 27:321-329

Moon RB, Richards JH (1973) Determination of intracellular $\mathrm{pH}$ by ${ }^{31} \mathrm{P}$ magnetic resonance. J Biol Chem 248:7276-7278

Moriyama S, Ishiwara T, Sakabe Y (1964) Studies on putrefaction of foods (IV): survey of putrefaction products of fish stored in the refrigerator, hot box or at constant temperature and room temperature. J Food Hyg Soc Jpn 5:361-365 (In Japanese with English abstract)

Murakami M, Okita Y, Matsuda H, Okino T, Yamaguchi K (1998) From the dinoflagellate Alexandrium hiranoi. Phytochemistry 48:85-88

Nicholson JK, Buckingham MJ, Sadler PJ (1983) High resolution ${ }^{1} \mathrm{H}$ NMR studies of vertebrate blood and plasma. Biochem J 211:605-615

Nicholson J, O’Flynn MP, Sadler P, Macleod A, Juul S, Sönksen P (1984) Proton-nuclear-magnetic-resonance studies of serum, plasma and urine from fasting normal and diabetic subjects. Biochem J 217:365-375

Ogata Y, Iwane R, Kimura I (2018) Properties of muscle protein of freeze-thawed olive flounder containing a high concentration of ATP when being frozen. Nippon Suisan Gakkaishi. https://doi. org/10.2331/suisan.18-00003

Ogawa S, Lee T-M, Kay AR, Tank DW (1990) Brain magnetic resonance imaging with contrast dependent on blood oxygenation. Proc Natl Acad Sci USA 87:9868-9872

Oike H, Nagai T, Furuyama A, Okada S, Aihara Y, Ishimaru Y, Marui T, Matsumoto I, Misaka T, Abe K (2007) Characterization of ligands for fish taste receptors. J Neurosci 27:5584-5592 
Paulus C, Rebets Y, Tokovenko B, Nadmid S, Terekhova LP, Myronovskyi M, Zotchev SB, Rückert C, Braig S, Zahler S (2017) New natural products identified by combined genomicsmetabolomics profiling of marine Streptomyces sp MP131-18. Sci Rep 7:42382

Peck LS, Pörtner HO, Hardewig I (2002) Metabolic demand, oxygen supply, and critical temperatures in the Antarctic bivalve Laternula elliptica. Physiol Biochem Zool 75:123-133

Pérez-Victoria I, Martín J, Reyes F (2016) Combined LC/UV/MS and NMR strategies for the dereplication of marine natural products. Planta Med 82:857-871

Pomin VH (2015) NMR structural determination of unique invertebrate glycosaminoglycans endowed with medical properties. Carbohydr Res 413:41-50

Porseryd T, Caspillo NR, Volkova K, Elabbas L, Källman T, Dinnétz P, Olsson P-E, Porsch-Hällström I (2018) Testis transcriptome alterations in zebrafish (Danio rerio) with reduced fertility due to developmental exposure to $17 \alpha$-ethinyl estradiol. Gen Comp Endocrinol 262:44-58

Purcell EM, Torrey HC, Pound RV (1946) Resonance absorption by nuclear magnetic moments in a solid. Phys Rev 69:37

Rabi II, Millman S, Kusch P, Zacharias JR (1939) The molecular beam resonance method for measuring nuclear magnetic moments. The magnetic moments of ${ }_{3} \mathrm{Li}^{6},{ }_{3} \mathrm{Li}^{7}$ and ${ }_{9} \mathrm{~F}^{19}$. Phys Rev 55:526

Rezzi S, Giani I, Héberger K, Axelson DE, Moretti VM, Reniero F, Guillou C (2007) Classification of gilthead sea bream (Sparus aurata) from ${ }^{1} \mathrm{H}$ NMR lipid profiling combined with principal component and linear discriminant analysis. J Agric Food Chem 55:9963-9968

Richards J, Heigenhauser G, Wood C (2003) Exercise and recovery metabolism in the pacific spiny dogfish (Squalus acanthias). J Comp Physiol B 173:463-474

Richards JG, Wang YS, Brauner CJ, Gonzalez RJ, Patrick ML, Schulte PM, Choppari-Gomes AR, Almeida-Val VM, Val AL (2007) Metabolic and ionoregulatory responses of the Amazonian cichlid, Astronotus ocellatus, to severe hypoxia. J Comp Physiol B 177:361-374

Ritola O, Lyytikäinen T, Pylkkö P, Mölsä H, Lindström-Seppä P (2000) Glutathione-dependent defence system and monooxygenase enzyme activities in Arctic charr Salvelinus alpinus (L.) exposed to ozone. Aquaculture 185:219-233

Rothman D, Behar K, Hetherington H, Den Hollander J, Bendall M, Petroff O, Shulman R (1985) ${ }^{1} \mathrm{H}$-Observe/ ${ }^{13} \mathrm{C}$-decouple spectroscopic measurements of lactate and glutamate in the rat brain in vivo. Proc Natl Acad Sci USA 82:1633-1637

Rothman DL, Behar KL, Hyder F, Shulman RG (2003) In vivo NMR studies of the glutamate neurotransmitter flux and neuroenergetics: implications for brain function. Annu Rev Physiol 65:401-427

Samuelsson LM, Larsson DJ (2008) Contributions from metabolomics to fish research. Mol BioSyst 4:974-979

Samuelsson LM, Förlin L, Karlsson G, Adolfsson-Erici M, Larsson DJ (2006) Using NMR metabolomics to identify responses of an environmental estrogen in blood plasma of fish. Aquat Toxicol 78:341-349

Santos EM, Ball JS, Williams TD, Wu H, Ortega F, Van Aerle R, Katsiadaki I, Falciani F, Viant MR, Chipman JK (2010) Identifying health impacts of exposure to copper using transcriptomics and metabolomics in a fish model. Environ Sci Technol 44:820-826

Sato W, Shikota R, Nozawa H (2011) Effects of storage under gaseous oxygen on degradation of trimethylamine-N-oxide in the muscle of Pacific saury. Nippon Suisan Gakkaishi 77:665-673 (In Japanese with English abstract)

Savorani F, Picone G, Badiani A, Fagioli P, Capozzi F, Engelsen SB (2010) Metabolic profiling and aquaculture differentiation of gilthead sea bream by ${ }^{1} \mathrm{H}$ NMR metabonomics. Food Chem 120:907-914

Segner H, Dölle A, Böhm R (1997) Ketone body metabolism in the carp Cyprinus carpio: biochemical and ${ }^{1} \mathrm{H}$ NMR spectroscopical analysis. Comp Biochem Physiol B Biochem Mol Biol 116:257-262

Seiler N, Al-Therib M, Kataoka K (1973) Formation of GABA from putrescine in the brain of fish (Salmo irideus Gibb.). J Neurochem 20:699-708

Shao Y, Li C, Chen X, Zhang P, Li Y, Li T, Jiang J (2015) Metabolomic responses of sea cucumber Apostichopus japonicus to thermal stresses. Aquaculture 435:390-397

Shen G, Huang Y, Dong J, Wang X, Cheng K-K, Feng J, Xu J, Ye J (2017) Metabolic effect of dietary taurine supplementation on Nile tilapia (Oreochromis nilotictus) evaluated by NMR-based metabolomics. J Agric Food Chem 66:368-377

Sheridan MA (1994) Regulation of lipid metabolism in poikilothermic vertebrates. Comp Biochem Physiol B Biochem Mol Biol 107B:495-508

Shikata T, Shimeno S (1997) Effects of feed restriction and starvation on fatty acid synthesis and oxidation of glucose and alanine in carp hepatopancreas. Fish Sci 63:301-303

Short CE, Driedzic WR (2018) Species-specific low plasma glucose in fish is associated with relatively high tissue glucose content and is inversely correlated with cardiac glycogen content. J Comp Physiol B 188:809-819

Shoubridge EA, Hochachka P (1980) Ethanol: novel end product of vertebrate anaerobic metabolism. Science 209:308-309

Shulman GI, Rothman D (2005) MRS studies of the role of the muscle glycogen synthesis pathway in the pathophysiology of type 2 diabetes. In: Shulman R, Rothman DL (eds) Metabolomics by in vivo NMR. Wiley, Chichester, pp 45-57

Southam AD, Easton JM, Stentiford GD, Ludwig C, Arvanitis TN, Viant MR (2008) Metabolic changes in flatfish hepatic tumours revealed by NMR-based metabolomics and metabolic correlation networks. J Proteom Res 7:5277-5285

Takada K (2015) Development of bioactive natural products from marine organisms. Nippon Suisan Gakkaishi 81:647-650 (In Japanese with English abstract)

Tokunaga T (1970) Trimethylamine oxide and its decomposition in the bloody muscle of fish. I. TMAO, TMA, and DMA contents in ordinary and bloody muscles. Nippon Suisan Gakkaishi 36:502509 (In Japanese with English abstract)

Traficante DD (1991) Time averaging. Does the noise really average towards zero? Concepts Magn Reson A 3:83-87

Treberg JR, Bystriansky JS, Driedzic WR (2005) Temperature effects on trimethylamine oxide accumulation and the relationship between plasma concentration and tissue levels in smelt (Osmerus mordax). J Exp Zool A Comp Exp Biol 303:283-293

Tsuda M, Kubota T, Sakuma Y, Ji Kobayashi (2001) Biosynthetic study of amphidinolide B. Chem Pharm Bull 49:1366-1367

Udwary DW, Zeigler L, Asolkar RN, Singan V, Lapidus A, Fenical W, Jensen PR, Moore BS (2007) Genome sequencing reveals complex secondary metabolome in the marine actinomycete Salinispora tropica. Proc Natl Acad Sci USA 104:10376-10381

Uemura D, Takahashi K, Yamamoto T, Katayama C, Tanaka J, Okumura Y, Hirata Y (1985) Norhalichondrin A: an antitumor polyether macrolide from a marine sponge. J Am Chem Soc 107:4796-4798

Valente ALS, Cuenca R, Zamora MA, Parga ML, Lavin S, Alegre F, Marco I (2006) Sectional anatomic and magnetic resonance imaging features of coelomic structures of loggerhead sea turtles. Am J Vet Res 67:1347-1353

van den Burg EH, Peeters RR, Verhoye M, Meek J, Flik G, van der Linden A (2005) Brain responses to ambient temperature 
fluctuations in fish: reduction of blood volume and initiation of a whole-body stress response. J Neurophysiol 93:2849-2855

van den Burg E, Verhoye M, Peeters R, Meek J, Flik G, van der Linden A (2006) Activation of a sensorimotor pathway in response to a water temperature drop in a teleost fish. J Exp Biol 209:2015-2024

van den Thillart G, van Waarde A (1996) Nuclear magnetic resonance spectroscopy of living systems: applications in comparative physiology. Physiol Rev 76:799-837

van den Thillart G, van Waarde A, Muller H, Erkelens C, Addink A, Lugtenburg J (1989) Fish muscle energy metabolism measured by in vivo ${ }^{31} \mathrm{P}-\mathrm{NMR}$ during anoxia and recovery. Am J Physiol Regul Integr Comp Physiol 256:R922-R929

van Eijsden P, Behar KL, Mason GF, Braun KP, de Graaf RA (2010) In vivo neurochemical profiling of rat brain by ${ }^{1} \mathrm{H}-\left[{ }^{13} \mathrm{C}\right] \mathrm{NMR}$ spectroscopy: cerebral energetics and glutamatergic/GABAergic neurotransmission. J Neurochem 112:24-33

van Ginneken V, van den Thillart G, Addink A, Erkelens C (1996) Synergistic effect of acidification and hypoxia: in vivo ${ }^{31} \mathrm{P}-\mathrm{NMR}$ and respirometric study in fishes. Am J Physiol Regul Integr Comp Physiol 271:R1746-R1752

van Ginneken V, van Den Thillart G, Muller H, van Deursen S, Onderwater M, Visee J, Hopmans V, Van Vliet G, Nicolay K (1999) Phosphorylation state of red and white muscle in tilapia during graded hypoxia: an in vivo ${ }^{31} \mathrm{P}-\mathrm{NMR}$ study. Am J Physiol Regul Integr Comp Physiol 277:R1501-R1512

van Waarde A, van den Thillart G, Erkelens C, Addink A, Lugtenburg J (1990) Functional coupling of glycolysis and phosphocreatine utilization in anoxic fish muscle. An in vivo ${ }^{31} \mathrm{P}$ NMR study. $\mathrm{J}$ Biol Chem 265:914-923

Viant M, Werner I, Rosenblum E, Gantner A, Tjeerdema R, Johnson M (2003) Correlation between heat-shock protein induction and reduced metabolic condition in juvenile steelhead trout (Oncorhynchus mykiss) chronically exposed to elevated temperature. Fish Physiol Biochem 29:159-171

Viant MR, Pincetich CA, Hinton DE, Tjeerdema RS (2006a) Toxic actions of dinoseb in medaka (Oryzias latipes) embryos as determined by in vivo ${ }^{31} \mathrm{P}$ NMR, HPLC-UV and ${ }^{1} \mathrm{H}$ NMR metabolomics. Aquat Toxicol 76:329-342

Viant MR, Pincetich CA, Tjeerdema RS (2006b) Metabolic effects of dinoseb, diazinon and esfenvalerate in eyed eggs and alevins of Chinook salmon (Oncorhynchus tshawytscha) determined by ${ }^{1} \mathrm{H}$ NMR metabolomics. Aquat Toxicol 77:359-371

Vieira VP, Inoue LAK, Moraes G (2005) Metabolic responses of matrinxã (Brycon cephalus) to dietary protein level. Comp Biochem Physiol A Mol Integr Physiol 140:337-342

von Brand E, Cisterna M, Merino G, Uribe E, Palma-Rojas C, Rosenblitt M, Albornoz JL (2009) Non-destructive method to study the internal anatomy of the Chilean scallop Argopecten purpuratus. J Shellfish Res 28:325-327

Vornanen M, Paajanen V (2006) Seasonal changes in glycogen content and $\mathrm{Na}^{+}-\mathrm{K}^{+}$-ATPase activity in the brain of crucian carp. Am J Physiol Regul Integr Comp Physiol 291:R1482-R1489

Wang I-N, Dykhuizen DE (2001) Variation of enzyme activities at a branched pathway involved in the utilization of gluconate in Escherichia coli. Evolution 55:897-908

Wang Y, Zhu W, Wang D, Teng M, Yan J, Miao J, Zhou Z (2017a) ${ }^{1} \mathrm{H}$ NMR-based metabolomics analysis of adult zebrafish (Danio rerio) after exposure to diniconazole as well as its bioaccumulation behavior. Chemosphere 168:1571-1577
Wang YW, Zhang JL, Jiao JG, Du XX, Limbu SM, Qiao F, Zhang ML, Li DL, Du ZY (2017b) Physiological and metabolic differences between visceral and subcutaneous adipose tissues in Nile tilapia (Oreochromis niloticus). Am J Physiol Regul Integr Comp Physiol 313:R608-R619

Watanabe T (1982) Lipid nutrition in fish. Comp Biochem Physiol B Comp Biochem 73:3-15

Wei Y, Liang M, Mai K, Zheng K, Xu H (2017) ${ }^{1}$ H NMR-based metabolomics studies on the effect of size-fractionated fish protein hydrolysate, fish meal and plant protein in diet for juvenile turbot (Scophthalmus maximus L.). Aquacult Nutr 23:523-536

Wilkie MP, Stecyk JA, Couturier CS, Sidhu S, Sandvik GK, Nilsson GE (2015) Reversible brain swelling in crucian carp (Carassius carassius) and goldfish (Carassius auratus) in response to high external ammonia and anoxia. Comp Biochem Physiol A Mol Integr Physiol 184:65-75

Williams TD, Davies IM, Wu H, Diab AM, Webster L, Viant MR, Chipman JK, Leaver MJ, George SG, Moffat CF (2014) Molecular responses of European flounder (Platichthys flesus) chronically exposed to contaminated estuarine sediments. Chemosphere 108:152-158

Wilson R (1994) Utilization of dietary carbohydrate by fish. Aquaculture 124:67-80

Wishart DS, Knox C, Guo AC, Eisner R, Young N, Gautam B, Hau DD, Psychogios N, Dong E, Bouatra S (2008) HMDB: a knowledgebase for the human metabolome. Nucleic Acids Res 37:D603-D610

Wu H, Southam AD, Hines A, Viant MR (2008) High-throughput tissue extraction protocol for NMR-and MS-based metabolomics. Anal Biochem 372:204-212

Wu JL, Zhang JL, Du XX, Shen YJ, Lao X, Zhang ML, Chen LQ, Du ZY (2015) Evaluation of the distribution of adipose tissues in fish using magnetic resonance imaging (MRI). Aquaculture 448:112-122

Yamamoto T, Unuma T, Akiyama T (2000) The influence of dietary protein sources on tissue free amino acid levels of fingerling rainbow trout. Fish Sci 66:310-320

Yamazaki M, Tachibana K, Satake M (2011) Complete ${ }^{13}$ C-labeling pattern of yessotoxin a marine ladder-frame polyether. Tetrahedron 67:877-880

Yoon SH, Itoh Y, Kaneko G, Nakaniwa M, Ohta M, Watabe S (2008) Molecular characterization of Japanese sillago vitellogenin and changes in its expression levels on exposure to 17 beta-estradiol and 4-tert-octylphenol. Mar Biotechnol 10:19-30

Young T, Alfaro AC (2018) Metabolomic strategies for aquaculture research: a primer. Rev Aquacult 10:26-56

Zang L-H, Howseman AM, Shulman RG (1991) Assignment of the ${ }^{1} \mathrm{H}$ chemical shifts of glycogen. Carbohydr Res 220:1-9

Zhang L, Liu X, You L, Zhou D, Yu J, Zhao J, Feng J, Wu H (2011) Toxicological effects induced by cadmium in gills of Manila clam Ruditapes philippinarum using NMR-based metabolomics. CLEAN Soil Air Water 39:989-995

Ziegler A, Faber C, Mueller S, Bartolomaeus T (2008) Systematic comparison and reconstruction of sea urchin (Echinoidea) internal anatomy: a novel approach using magnetic resonance imaging. BMC Biol 6:33

Ziegler A, Kunth M, Mueller S, Bock C, Pohmann R, Schröder L, Faber C, Giribet G (2011) Application of magnetic resonance imaging in zoology. Zoomorphology 130:227-254 